\title{
CHLORINATED LIPID INDUCE INFLAMMATORY RESPONSES IN THE MICROCIRCULATION
}

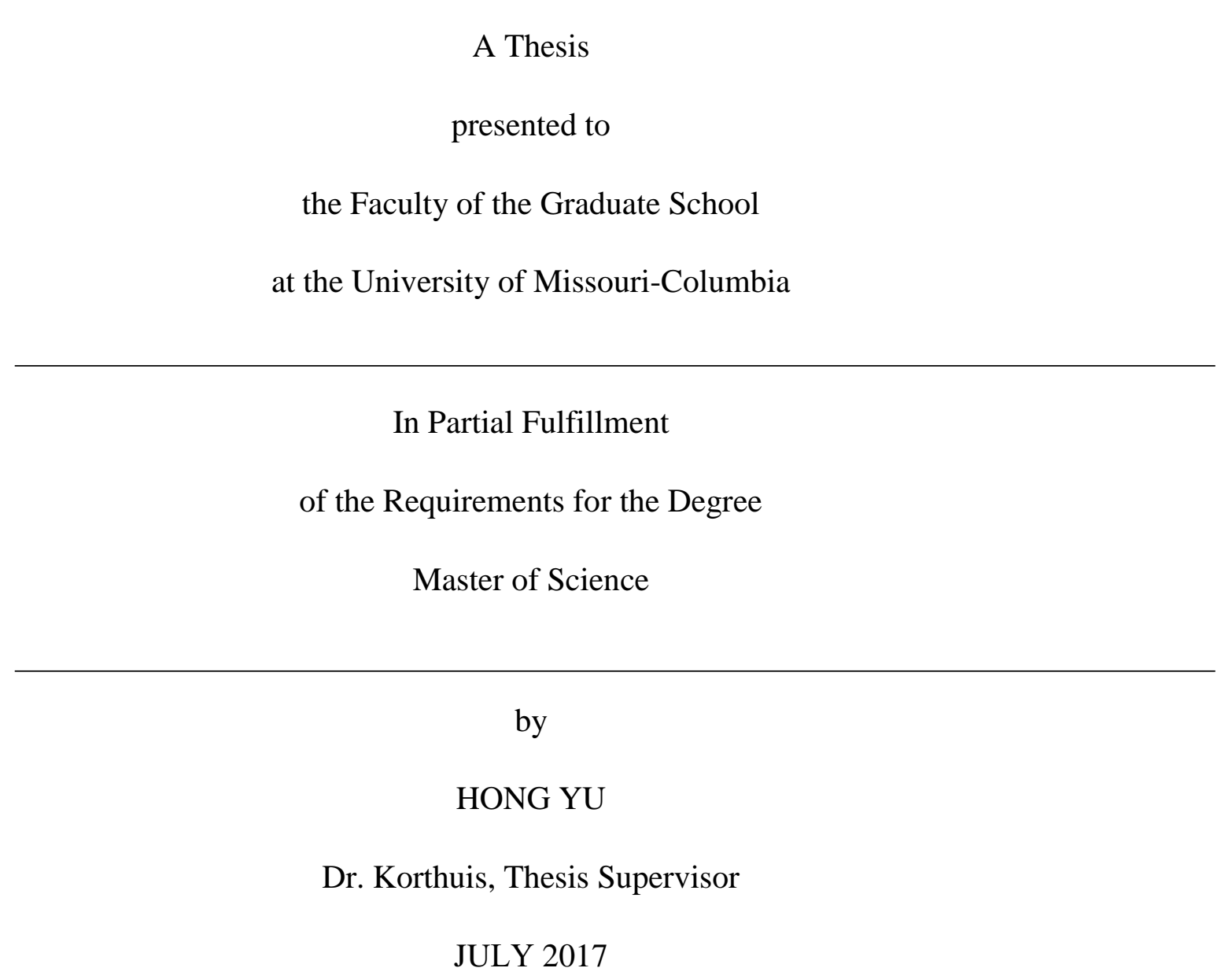


The undersigned, appointed by the dean of the Graduate School, have examined the thesis entitled

\section{CHLORINATED LIPID INDUCE INFLAMMATORY RESPONSES IN THE}

\section{MICROCIRCULATION}

presented by Hong Yu,

a candidate for the degree of Master of Science,

and hereby certify that, in their opinion, it is worthy of acceptance.

Professor Ronald J. Korthuis

Professor David A. Ford

Professor Alan Parrish 


\section{ACKNOWLEDGEMENTS}

I would first like to thank my thesis advisor Professor Ronald J. Korthuis for his patient mentoring and constant support. The door to Dr. Korthuis office was always open whenever I ran into a trouble spot or had a question about my research. He consistently allowed this project to be my own work, prompted me fully focus on my scientific pursuits and always kept me pointed in the right direction. I am grateful to have such an approachable, passionate and experienced mentor providing oversight of my studies and experimentation.

I would also like to thank Professor David A. Ford for his constructive comments and insights on experimental design, implementation and analysis of experimental results. Many thanks are due Professor Alan Parrish for giving valuable suggestions on my experimental design.

So too, I thank the experts who were involved in this project: Laboratory Manager Meifang Wang and Assistant Research Professor Theodore J. Kalogeris. Without their sustaining support, this project could not have been successfully conducted. Thanks to my fellow lab member Mike Birkhead for his very valuable input towards my experiments and making the life in the laboratory a true pleasure. 


\section{TABLE OF CONTENTS}

ACKNOWLEDGEMENTS.....................................................

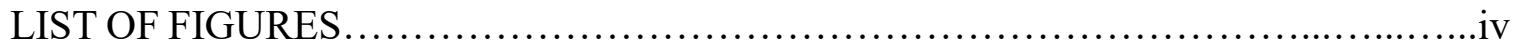

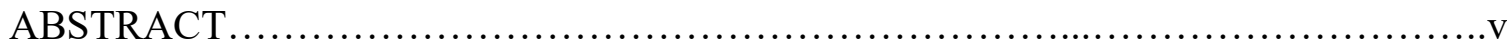

INTRODUCTION

Preface....................................................................

Neutrophil-MPO Derived Chlorinated lipid.......................................

Biological Effects of Chlorinated lipid.....................................6

Microcirculatory Dysfunction................................................. 8

\section{MATERIALS AND METHODS}

Experimental Animals.................................................. 16

Materials.................................................................. 16

Experimental Protocol................................................. 16

Intravital Microscopy................................................... 18

MPO Activity Analysis.....................................................20

Statistical Analysis.................................................... 22

RESULTS........................................................... 23

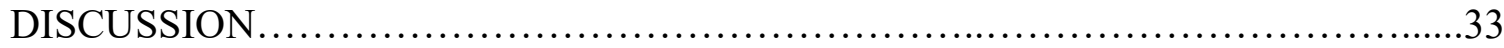

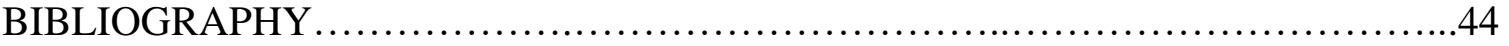




\section{LIST OF FIGURES AND TABLES}

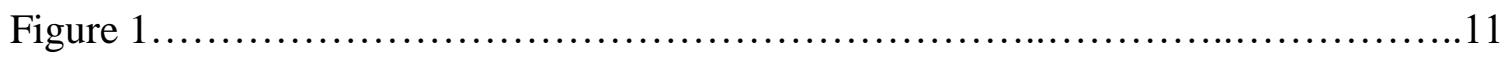

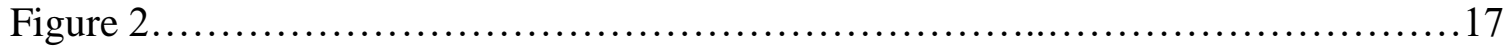

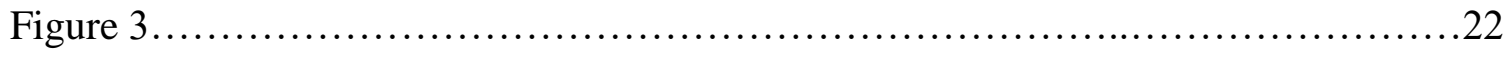

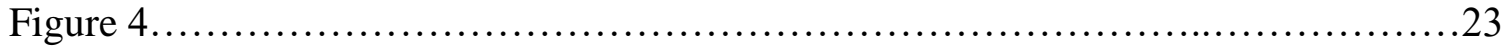

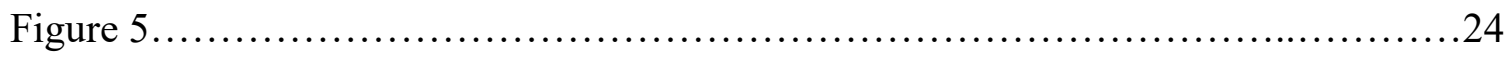

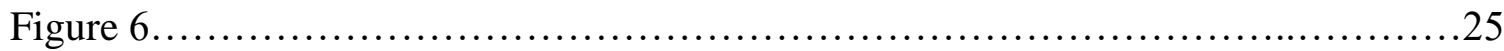

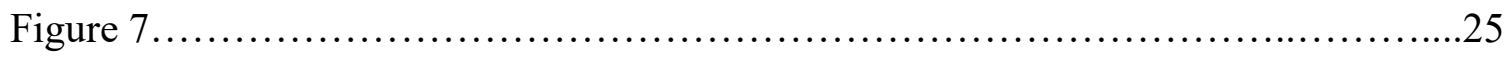

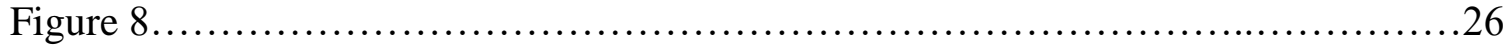

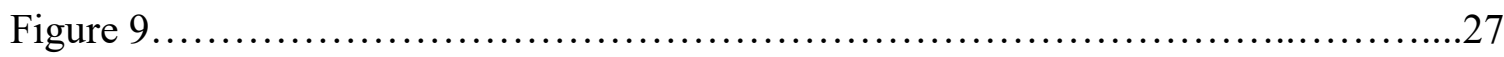

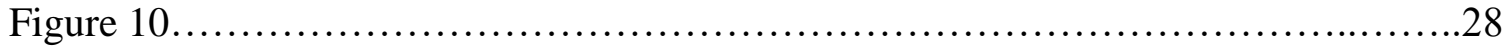

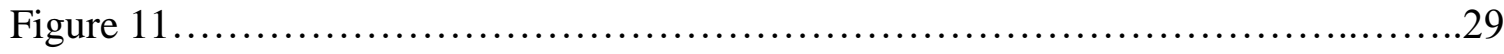

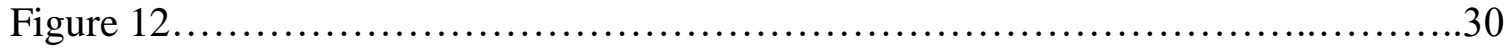

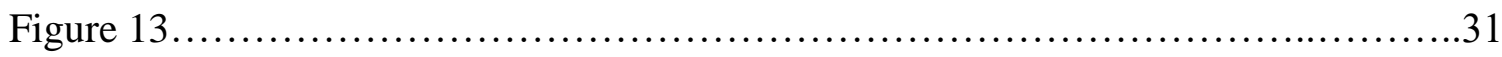

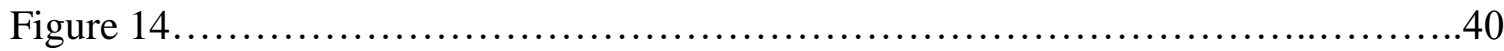

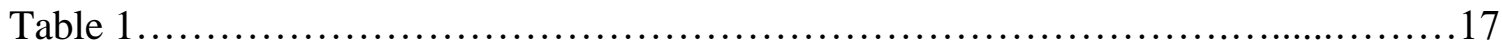




\title{
CHLORINATED LIPID INDUCE INFLAMMATORY RESPONSES IN THE MICROCIRCULATION
}

\section{Hong Yu}

\section{Ronald J. Korthuis Thesis Supervisor}

\begin{abstract}
Previous studies from our research group have shown that chlorinated lipid, generated from the neutrophil-myeloperoxidase (MPO) system, are elevated in a rat sepsis model as well as in plasma of septic patients. Other work reported in multiple experimental studies and human trials have shown that microcirculatory dysfunction is a hallmark of sepsis. However, whether and how chlorinated lipid contribute to microcirculatory dysfunction are still unclear. In the current study, we hypothesized that compared to non-chlorinated lipid, chlorinated lipid could elicit inflammatory responses in the microcirculation.

To test this postulate, a specific rat intravital model was developed. Briefly, male Sprague Dawley rats (250-300g) were randomly divided into 4 groups: 2-chloropalmitic acid (2-ClPA) group, 2-chloropalmitaldehyde (2-ClHDA) group, non-chlorinated palmitic acid (PA) group and palmitaldehyde (HDA) group $(n=6)$. Rat mesenteries were exteriorized and superfused with $10 \mu \mathrm{M}$ of 2-CIPA or 2-ClHDA, respectively. Equimolar concentrations of PA and HDA were applied as controls. Via use of our intravital microscopic approach, the indicators of microcirculatory dysfunction, leukocyteendothelial interactions (leukocyte rolling and adhesion), mast cell degranulation, reactive oxygen species (ROS) production, and albumin leakage were evaluated at 0 min (baseline),
\end{abstract}


$20 \mathrm{~min}, 50 \mathrm{~min}$ and $80 \mathrm{~min}$ after the initiation of lipid superfusion, respectively. At the end of experiments, rats were sacrificed and the jejunum was collected. MPO expression from granulocytes of the jejunum was assessed by a fluorescence assay and immunohistochemistry (IHC) staining.

Results of this study showed: (1) In the PA group, very few rolling and adhesive leukocytes were detected, while in the 2-ClPA treated group, there was a significant increase in rolling and adhesive leukocytes. At all the time points examined, 2-ClPA produced significant increases in mast cell degranulation, ROS production and albumin leakage, compared with PA. (2) In the HDA group, no increase in leukocyte rolling and adhesion were observed, while 2-ClHDA produced an increase in leukocyte rolling but not stationary adhesion. HDA produced a time-dependent increase in mast cell degranulation, but this was lower than that seen in response to 2-ClHDA. ROS production and albumin leakage were significantly lower in response to HDA than with 2-ClHDA. (3) MPO expression in rat jejunum was almost 2 times higher in 2-CIPA and 2-ClHDA treated groups compared with PA and HDA groups, respectively. Similarly, the IHC staining data showed that MPO expression in 2-ClPA and 2-ClHDA treated groups was more than 3fold higher than in PA and HDA groups, respectively.

In summary, our data indicate that chlorinated lipid, 2-ClPA and 2-ClHDA, elicit inflammatory responses in rat mesentery, which are characterized by elevated leukocyteendothelial interactions (leukocyte rolling and adhesion), mast cell degranulation, ROS production, and endothelial barrier disruption (albumin leakage). These changes were associated with increased MPO expression level in jejunum submucosa of intestinal segments adjacent to the superfused mesentery in response to the two chlorinated lipid. 
These inflammatory responses mimic those produced by sepsis, thereby providing evidence supporting the hypothesis that chlorinated lipid, generated from the enzymatic activity of neutrophil-MPO system, induce microcirculatory dysfunction via stimulating inflammatory responses when compared to non-chlorinated lipid. In future studies, we propose to directly test this postulate by applying MPO inhibitors to septic models, and monitoring whether chlorinated lipid production would be reduced and the aforementioned inflammatory responses can be attenuated. 


\section{INTRODUCTION}

\section{Preface}

Sepsis is a dangerous immune response to an infection striking adults and children of every age. The Centers for Disease Control and Prevention estimate that over a million people are hospitalized for sepsis each year, and is a major cause of morbidity and mortality, especially in older and immunocompromised patients. The necessity of early identification of sepsis lends urgency to the investigation of the pathophysiology of the disease and to identify reliable biomarkers. In sepsis, activated leukocytes, in particular polymorphonuclear neutrophils (PMNs), release an important antimicrobial enzyme, myeloperoxidase (MPO), which catalyzes the formation of hypochlorous acid ( $\mathrm{HOCl})$ from hydrogen peroxide $\left(\mathrm{H}_{2} \mathrm{O}_{2}\right)$ and chloride $(1 ; 2)$. Neutrophil-MPO system induces reactive chlorinated species (RCS) production, which can protect the host against invading microbes physiologically while exerting oxidative effects on plasmalogens pathologically. It has been demonstrated that 2-chlorohexadecanal (or 2-chloropalmitaldehyde, 2-ClHDA) and other chlorofatty aldehydes are produced when neutrophils are activated via the production of MPO-derived RCS that attack both neutrophil and endothelium plasmalogen pools (3). Once in the tissue, it is possible that parenchymal cells can be attacked in the same way by resident neutrophils activation, thereby exacerbating injury. Furthermore, as an intermediate of the fatty acid-fatty alcohol cycle, 2-ClHDA can be metabolized into 2chlorohexadecanoic acid (or 2-chloropalmitic acid, 2-CIPA) (4). 
In sepsis, microcirculatory dysfunction is frequent, manifesting as disruption in the ability of the microcirculation to maintain adequate oxygen release and the delivery of other nutrients to tissues as well as impaired removal of metabolic byproducts, this leads to parenchymal cell death. The severity of microvascular alterations is associated with organ dysfunction and mortality. The reported main mechanisms of the development of microcirculatory dysfunction involves inflammatory responses, which includes endothelial dysfunction, impaired cell-cell communication, adhesion, rolling and emigration of white blood cells, mast cell degranulation, reactive oxygen species (ROS) production, and endothelial barrier disruption (5).

Of note, initial investigations from our collaborative group showed that 2-chlorofatty acid (2-ClFA) levels in plasma were significantly greater in patients with sepsis and in a rat cecal ligation and puncture sepsis model. In vitro, human endothelial cells treated with 2-ClFA species, including 2-ClPA and 2-ClHDA, demonstrated increased endothelial surface expression of P-selectin, E-selectin, vascular cell adhesion protein 1 (VCAM-1) and intercellular adhesion molecular 1 (ICAM-1). Moreover, with application of 2-CIPA and 2-ClHDA, human microvessel endothelial cells from lung and mesentery showed reduced endothelial barrier function (6). These data suggested that the chlorinated lipid likely play a role in the development of microcirculatory dysfunction by directly influencing the inflammatory response in sepsis patients. Therefore, the goal of this study was to further characterize microcirculatory dysfunction induced by chlorinated lipid, 2ClPA and 2-ClHDA, via quantifying their effects on inflammatory response including leukocyte/endothelial interactions (leukocyte rolling and adhesion), mast cell degranulation, ROS production, endothelial barrier dysfunction (albumin leakage), and the 
expression level of MPO (a chlorinated lipid-generating enzyme released by neutrophils) in a rat sepsis model. This will help us better understand the function of chlorinated lipid involved in the microcirculatory dysfunction, and the possibility that these chlorinated lipid may provide reliable biochemical markers for the diagnosis of sepsis patients.

\section{Myeloperoxidase Derived Chlorinated Lipid Species}

Myeloperoxidase (MPO) is an important antimicrobial enzyme in azurophilic granules of phagocytes, and is especially enriched in neutrophils (7). Neutrophils are critical in bacterial killing and in clearance of invading microbes during inflammation, but these cells can also damage host cells when activated. In the process of neutrophil activation, MPO plays a central role in inducing the production of neutrophil-derived cytotoxic species that contribute to cell killing $(8 ; 9)$. The basic function of MPO is to catalyze the interaction of $\mathrm{H}_{2} \mathrm{O}_{2}$ and chloride, to produce $\mathrm{H}_{2} \mathrm{O}_{2}$ to hypochlorous acid (HOCl) (10).

Due to its chemical reactivity, $\mathrm{HOCl}$ has been shown to exert microbicidal and cytotoxic activity $(11 ; 12)$. Thus $\mathrm{HOCl}$ reacting with lipids could give rise to chlorination

of amines, alkenes and vinyl ethers $(13 ; 14)$. Besides of $\mathrm{HOCl}$, conjugate base $\left(\mathrm{OCl}^{-}\right)$of $\mathrm{HOCl}$ and chlorine gas $\left(\mathrm{Cl}_{2}\right)$ produced by neutrophil-MPO system, comprise the major reactive chlorinating species $(\mathrm{RCS})(10 ; 15)$. In equilibrium with $\mathrm{OCl}^{-}$and $\mathrm{Cl}_{2}, \mathrm{HOCl}$ mediates several critical reactions related to cytotoxic properties of activated neutrophils, by the mechanisms of oxidative bleaching of protein heme and iron sulfur centers, in conjunction with primary amine groups and the generation of oxidizing chloramines (16; 17). RCS target alkene groups $(-\mathrm{C}=\mathrm{C}-)$ of cholesterol or unsaturated fatty acids in 
generation of chlorohydrins (18). It is possible that chlorohydrins and other chlorinated lipid-derived products partially mediate the cytotoxicity attributed to RCS $(19 ; 20)$.

In human heart tissue, nearly $30-40 \%$ of choline glycerophospholipids are plasmalogens, which implies that plasmalogens are a predominant phospholipid present in plasma membrane in cardiovascular system (21-23). In plasma membrane, plasmalogens are highly enriched in lipid rafts $(24 ; 25)$. Endothelial cell membranes are enriched with plasmalogens while circulating cells such as neutrophils and monocytes also have highly abundant pools of plasmalogens $(26 ; 27)$. Plasmalogen is characterized by the presentence of a vinyl ether linkage especially at $s n-1$ position, which is typically derived from $\mathrm{C} 16: 0$, C18:0, or C18:1 fatty alcohols (21-23). The vinyl ether linkage at sn-1 is acid labile and a masked aldehyde. Normally, the masked aldehyde is relatively inert enzymatically, while being accessible in the hydrophilic plane of cell membrane bilayers where it can be targeted by oxidants. It has been demonstrated that plasmalogens protect mammalian cells from ROS attacking through putative anti-oxidant properties (21-23). On the other hand, it was shown that in a cell free system, plasmalogen is targeted by RCS, especially $\mathrm{HOCl}$ derived from MPO in activated leukocytes, and a neutral lipid released was identified as $\alpha$ chlorofatty aldehyde (3). In plasmalogens, such as plasmenylcholine, the sn-1aliphatic chain is attached to the glycerol backbone by a vinyl ether bond, which makes them different from the phospholipids containing esterified fatty acids. $\mathrm{HOCl}$ can easily attack to sn-1vinyl ether bond and yield a lysolipid and $\alpha$-chlorofatty aldehydes (3). To be specific, 2-chlorohexadecanal (2-ClHDA) is formed by neutrophil-MPO-HOCl system attacking on the plasmalogen 1-O-hexadec-1'-enyl-2-acyl-sn-glycero-3-phosphocholine, while 2-chlorooctadecanal (2-ClOA) is by 1-O-octadec-1'-enyl-2-acyl-sn-glycero-3- 
phosphocholine; in neutrophils activated with PMA 2-ClOA and 2-ClHDA have been detected (18).

The reports of chlorinated fatty acids and other lipids go back to at least the 1970s and the organohalogen compounds are widely demonstrated in biologic systems (28). However, halogenated compounds are considerably rarer in mammals, even though MPO and other haloperoxidases are present $(29 ; 30)$. The formation of halogenated lipids in mammalian systems is not considered to be a normal physiological process but rather represents undesirable and damaging molecules formed in adverse conditions. Although some chlorinated derivatives are produced naturally in animals, in mammals the majority of chlorinated lipid are thought to be induced by oxidative damage in the process of inflammation via the action of halogenating enzymes such as MPO. To identify chlorinated lipid derived from phospholipids, a number of methods have been applied: 1) thin-layer chromatography (TLC) is applied to separate $\alpha$-chlorofatty aldehydes from other chlorinated species or non-chlorinated lipid with comparison specific retention factors with synthesized standards (31); 2) gas chromatography-mass spectrometry (GCMS) has been extensively used for the analysis of different types of chlorinated lipid and confirms the formation of 2-ClHDA. The $\alpha$-chlorofatty aldehydes, especially the 2-ClHDA, are produced via RCS, which in turn are generated by MPO in activated neutrophils, attacking both neutrophil and endothelial cell plasmalogen pools (18). However, it is important to determine whether this 2-ClHDA is of biological relevance or metabolized to functionally relevant molecules. 2-ClHDA Schiff base adducts have been identified and other studies exploring of 2-ClHDA metabolism show that neutrophils and endothelial cells can metabolize 2-ClHDA to 2-chlorohexadecanol ( $\alpha$-chlorofatty alcohol) and 2- 
chlorohexadecanoic acid ( $\alpha$-chlorofatty acid, 2-CIPA) (4). Despite that it could be metabolized within cells, the metabolites of 2-ClHDA were actively secreted outside of cells instead of accumulating intracellularly. At the same time, 2-ClPA can be incorporated by esterification into complex lipids such as triglycerides and phospholipids. In addition, a cell culture model of fatty aldehyde dehydrogenase (FALDH) deficiency indicated that FALDH was essential for conversion of 2-ClHDA to 2-ClPA. Neutrophils oxidize on reduce endogenously 2-ClHDA to 2-ClPA or 2-chlorofatty alcohol respectively (32). In conclusion, plasmalogen targeting by MPO-derived $\mathrm{HOCl}$ induces not only 2-ClHDA but also a family of chlorinated lipid including 2-ClPA and 2-chlorofatty alcohol (2-ClFOH).

\section{Biological Effects of Chlorinated lipid}

Important points to consider regarding the biologic relevance of chlorinated lipid is under what conditions and concentrations they might be produced. The possible production of chlorinated lipid and chlorinated fatty acids, resulting from MPO activity in the process of inflammatory conditions such as sepsis or $\mathrm{I} / \mathrm{R}$, are the main focus. To date, evidence supporting the formation of chlorinated lipid in vivo is circumstantial, with the exception being $\alpha$-chlorofatty aldehydes. The $\alpha$-chlorofatty aldehydes ( $\alpha$-ClFALD) contribute to tissue injury by the following mechanisms: 1) inhibiting eNOS (20); 2) activating NFkB pathway and eliciting endothelial dysfunction (33; 34); 3) promoting neutrophil chemotaxis (18); 4) eliciting myocardial contractile dysfunction; and 5) neuronal apoptosis $(17 ; 19)$. However, their roles in relevant pathologic conditions have not been widely evaluated.

In contrast to phospholipid chlorohydrins and chlorinated sterols, an increasing body of evidence shows that $\alpha$-chlorofatty aldehydes are produced in vivo and increased in some 
forms of cardiovascular disease. For one thing, in human monocytes treated with MPO or PMA, 2-ClHDA and 2-chlorooctadecanal (2-ClODA) were generated from LDL plasmalogens (35). Other work has investigated the role of 2-ClHDA in human atherosclerotic plaque samples, and these studies showed that 2-ClHDA levels increased by almost 1400 fold compared with control artery samples (36). Interestingly, 2-ClHDA accumulated in rat hearts after myocardial infarction induced by coronary artery occlusion, whereas levels were decreased in neutropenic rats. The latter observation implied that the occurrence of 2-ClHDA was dependent on neutrophil infiltration (17). However, none of these findings demonstrates a cause and effect relationship with regard to pathologic mechanisms.

So far, the main focus of research in this area have been to demonstrate potential effects of chlorinated lipid that might contribute to disease. For example, fatty acid exposure caused depletion of cellular ATP with loss of cell viability $(37 ; 38)$. Moreover, low micromolar concentrations of 2-ClHDA $(2-10 \mu \mathrm{M})$ have been found to decrease eNOS expression in human umbilical cord endothelial cells and inhibit nitric oxide production, effects that could induce proinflammatory responses. Treatment with exogenous 2-ClHDA at the concentrations of $10-20 \mu \mathrm{M}$ adversely affected the performance of Langendorffperfused rat hearts, as evidenced by decreased heart rate, and toxicity as assessed by elevated lactate dehydrogenase release (17). To sum up, the 2-ClHDA concentrations reach $90 \mu \mathrm{M}$ in phorbol ester-stimulated human neutrophils while levels estimated to be $1 \mu \mathrm{M}$ occur in infarcted rat myocardium $(17 ; 18)$. In plasma and phorbol ester-stimulated neutrophils, levels of 2-ClHDA were noted at $1 \mathrm{nM}$ and $10 \mu \mathrm{M}$, respectively (32). Of note, in human atherosclerotic lesions, 2-ClHDA levels of approximately $10 \mu \mathrm{M}$ have been 
calculated, which is based on $2.08 \mathrm{pmol} 2-\mathrm{ClHDA} / \mathrm{nmol}$ lipid phosphorus and $3400 \mathrm{nmol}$ lipid phosphorus/g wet tissue and the assumptions that $75 \%$ of the wet weight is water and 2-ClHDA has free access to all water space (36). The chemoattractive activity of 2-ClHDA has been observed at both nanomolar and micromolar levels. Thus, it is reasonable to assume that 2-ClHDA at the concentration of $10 \mu \mathrm{M}$ in vivo could be a phagocyte chemoattractant (6). Recent studies showed that as one component of hypochloritemodified LDL, 2-ClHDA induced apoptosis that was mediated by ROS in Jurkat T cells $(27 ; 35 ; 39)$. Another study reported that, via the fatty alcohol-fatty acid cycle, 2-ClHDA forms a Schiff base and was oxidized to 2-chlorinated fatty acid, such as 2-CIPA, which leads to $\mathrm{H}_{2} \mathrm{O}_{2}$ production from THP-1 monocytes (40).

\section{Inflammatory Response in Microcirculation Dysfunction}

\section{Chlorinated lipid are Potential Endogenous Pro-Inflammatory Molecules}

The microcirculation, comprised of vessels less than 100-300 $\mu \mathrm{m}$ in diameter including arterioles, venules and capillaries, with its endothelium represents the largest organ in the body (6). Main cellular components in microcirculation are endothelial cells, and smooth muscle cells (41). The microcirculation in all tissues performs essential homeostatic functions including oxygen delivery and solute exchange (41). The development of microvascular dysfunction involves endothelial dysfunction, endothelial barrier disruption as well as diminished production of gaseous signaling molecules, cellcell communication dysfunction, mast cell degranulation, and the production of ROS and pro-inflammatory mediators. 
Microcirculatory functions critical for the homeostatic control of infection can become dysregulated and harmful during sepsis. Much evidence suggests that the defect of sepsis is not a loss of macrovascular blood supply but rather a loss of microvascular function, which has been identified as critical pathogenic endothelial barrier dysfunction, inappropriate neutrophil/endothelial cell interactions, ROS production and mast cell degranulation $(42 ; 43)$. Initial investigations from our collaborative group showed that in septic patients, 2-chlorofatty acid (2-CIFA) levels were significantly greater in comparison to healthy humans. Similar observations were noted in a preclinical study with rat model of cecal ligation and puncture, wherein 2-ClFA level in plasma and cecum were both elevated compared to those with sham surgeries. Further studies conducted in cultured human endothelial cells demonstrated increased cell surface expression of P-selectin, Eselectin, VCAM-1 and ICAM-1 following treatment with 2-ClHDA and 2-CIPA respectively, in comparison with either vehicle or equimolar HDA and PA. Moreover, human microvessel endothelial cells exposed to 2-ClHDA or 2-ClPA demonstrated increased endothelium cortical stiffness coupled with reduced endothelial barrier function. These observations suggested that in sepsis the potential for abundant production of chlorinated lipid at the neutrophil-endothelial interface make it possible that chlorinated lipid could be the factor mediating endothelial/microcirculatory dysfunction and eliciting inflammatory responses in vivo.

Endothelial cells, which line microvasculature and macrovessels, play a central role in control of microcirculation, regulating leukocyte-endothelial cell interactions, leukocyte migration, platelet adhesion/aggregation, trafficking of nutrients and capillary permeability (44). Under physiological conditions, endothelial cells sense metabolic changes and 
respond by regulating local blood flow via release of nitric oxide (NO), adjusting smooth muscle tone in arterioles. As an activator of soluble guanylate cyclase enzyme, NO induces the production of cGMP, which mediates smooth muscle cell relaxation. Whereas endothelial cells are activated as part of the inflammatory response to tissue injury, with upregulation of inducible nitric oxide synthase (iNOS) and endothelin-1 (45; 46), the NO system is severely affected in sepsis. On one hand, iNOS is expressed heterogeneously, resulting in pathological shunt of microvascular blood supply. On the other hand, endothelial nitric oxide synthase (eNOS) is affected by the reactive oxygen species (ROS), leading to interference of $\mathrm{NO}$ formation and bioavailability. In addition, activated neutrophils can secrete proteolytic enzymes, including elastase, matrix metalloproteinases (MMPs), and oxygen radicals with short-term and long-term effects that produce endothelial dysfunction (47). Endothelial cells also lose regulatory function, contributing to abnormalities in microcirculation in many other ways: loss of coupling with smooth muscle cells, loss of electrophysiological communication between endothelial cells, and changes in signal transduction pathways $(41 ; 48-50)$.

\section{Neutrophil-Endothelial Cells Interactions in Inflammatory Response}

The recruitment of neutrophils towards local inflammatory stimuli occurs via several steps (Figure 1): margination, capture (tethering), rolling, slow rolling, arrest (firm adhesion), luminal crawling, transendothelial migration, abluminal crawling and penetration of the basement membrane at low matrix protein deposition regions and through pericyte gaps, detachment and eventually emigration into interstitium (51). The neutrophil-endothelium interactions, mainly including leukocyte rolling, adhesion and transmigration across microvascular endothelium, occur primarily in postcapillary venules. 


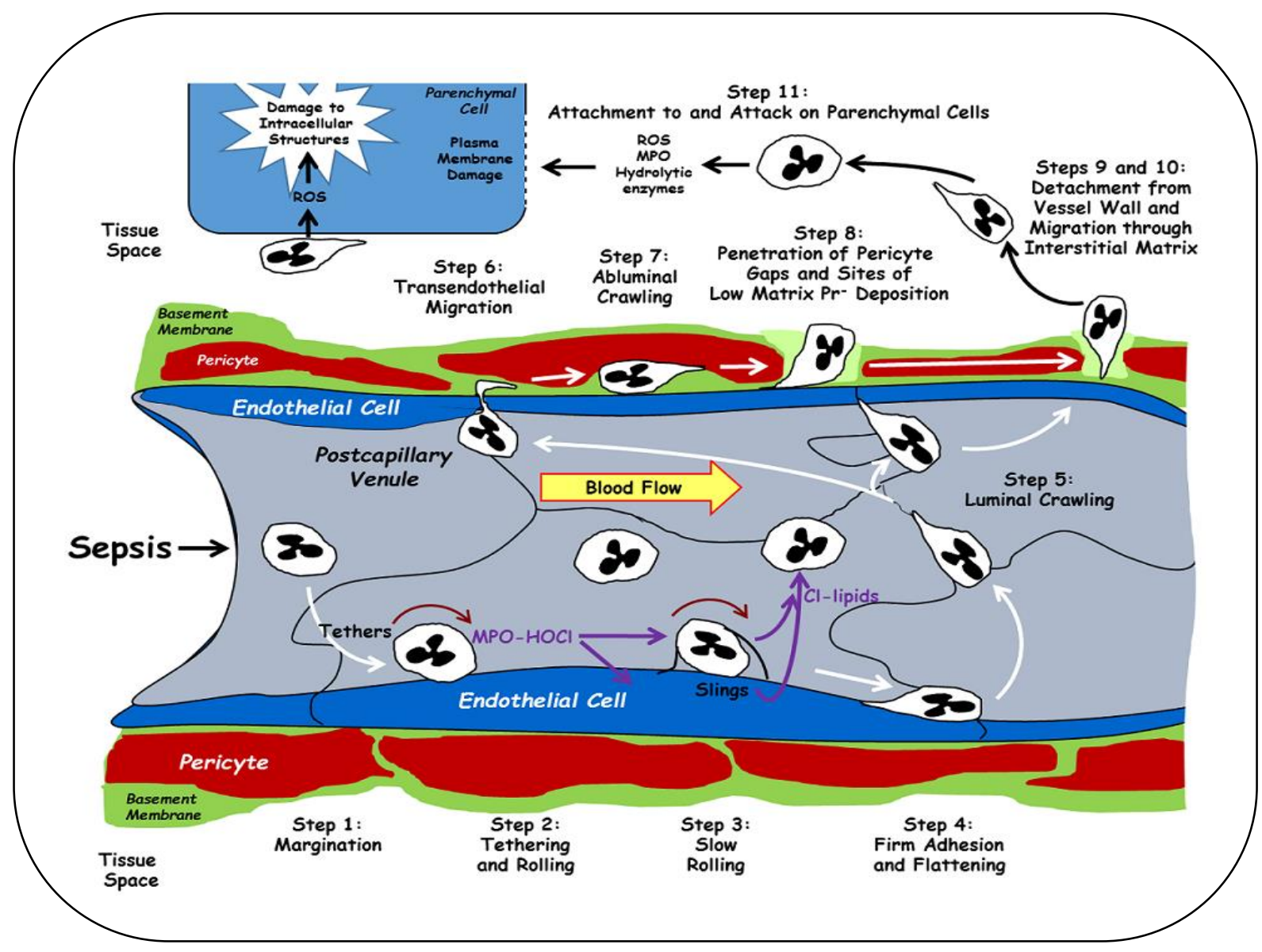

Figure 1. Neutrophils-endothelial cells interactions and MPO-HOCl-Chlorinated lipid system. Image courtesy of Zuidema MY, University of Missouri, Missouri, USA

Several well-known structures exert effects on neutrophil-endothelial cell interactions, such as adhesion molecule expression on the surface of leukocytes and/or endothelial cells, electrostatic charge interactions between surface structures on both cells, and hydrodynamic dispersal forces that promote leukocyte margination while shear stress imposed by flowing blood tends to sweep these cells away from the vessel wall. In the first place, neutrophils exit capillaries and marginate to postcapillary venular wall under the guidance of hydrodynamic dispersal forces. Therefore, when adhesion molecule or 
receptors are expressed on both cells, neutrophils tether to and roll along endothelium surfaces on condition that the tethering force exceeds shear forces, which can sweep nonactivated neutrophils away from non-inflamed endothelial cells.

It is worth noting that adhesive molecules, including specific integrin members of immunoglobin superfamily, selectins and some other molecular entities play an import role in neutrophil/endothelial cell interactions (51). To be specific, E-selectin and P-selectin expressed on activated endothelial cells interact with L-selectin and P-selectin glycoprotein ligand 1 (PSGL-1) on neutrophils to mediate leukocyte rolling and adhesion. For Eselectin, it takes 2-4 h for de novo synthesis; whereas P-selectin can move to cell surface directly from a stored pool in Weibel-Palade bodies, following by newly synthesized Pselectin 1-4 $\mathrm{h}$ later. Thus, P-selectin is responsible for the early phase of leukocyte rolling while newly synthesized selectins mediate longer term rolling. Rolling leukocytes become activated secondary to releases of chemoattractant and intracellular signals during the rolling phase. The binding of integrin $\mathrm{CD} 11 \mathrm{a} / \mathrm{CD} 18$ on activated leukocytes surface with its corresponding receptor ICAM-1 on endothelial cells, works to strengthen neutrophil/endothelium interactions (51). Through the CD11b/CD18-ICAM-1 binding, neutrophils start to crawl along the vessel lumen in search of regions along the barrier for diapedesis. Leukocytes normally transmigrate wherever the underlying basement has low matrix protein deposition and usually the endothelial junction with enriched ICAM-1 expression. In general, diapedesis via intercellular junctions involves junctional proteins, such as junctional molecule A and C (JAM-A and JAM-C) interacting with adhesive molecules CD11a and CD11b on neutrophils. After penetration, neutrophils move away from endothelial barrier towards pericytes via CD11a/CD11b/ICAM-1 binding, and then 
crowd towards basement membrane through very late antigen (VLA-3/VLA-6) adhesion molecule mechanism. The neutrophils preferentially crawl over a gap in pericytes and low matrix protein area in basement membrane to detach the vessel structure (52-54).

\section{Neutrophil, Mast Cell and ROS in Inflammatory Responses}

2-ClHDA, which is a neutrophil chemoattractant, could enhance the recruitment of neutrophils to areas of active inflammation (3). As a critical first responder of the innate immune response, neutrophils are activated to kill pathogens via phagocytosis, generation of reactive oxygen and nitrogen species, release peptides and proteases and other hydrolytic enzymes to extracellular space, as well as the formation of neutrophil extracellular traps (NETs) (55). NETs, the end-product of this distinct form of programmed cell death termed "NETosis", are networks of extracellular fibers, primarily composed of DNA from neutrophils, which bind pathogens (56-58). The neutrophil-MPO system could induce chlorinated lipid generation via plasmalogen oxidation, whereas $\mathrm{HOCl}$ is a $\mathrm{ROS}$, generated from neutrophil-MPO system, that is involved in NETosis (59). In addition, mast cell derived tumor necrosis factor alpha (TNF $\alpha$ ) plays an important role in inflammation through the recruitment and infiltration of neutrophils (60).

Mast cells can respond to any harmful tissue injury by degranulation, which releases a large amount of proinflammatory stimuli, providing protection against a wide spectrum of microorganisms, affecting primarily the permeability of vessels by slow release of inflammatory mediators and chemotactic factors in the process of degranulation $(61 ; 62)$. Secreted tryptases and chymases, such as cathepsin G, promote inflammation, matrix destruction and tissue remodeling by several mechanisms. However, the tryptase and chymases can also oppose inflammation by inactivation allergens and neuropeptides. 
Therefore, beneficial or harmful roles of mast cells and their relative serine peptidases could be context-specific (63).

ROS are oxidants derived from oxygen molecules having one or more unpaired electrons in atomic or molecular orbitals, which makes them highly reactive. In high concentrations, ROS exert damaging effects on DNA, lipids and proteins; however, at low concentrations ROS can cause reversible posttranslational modifications of proteins involved in intracellular signal transduction pathways (64-67). Interestingly, ROS play an important role in FceRI-dependent signaling that provides rapid degranulation of mast cells (68; 69), and induce NETosis via the neutrophil-MPO system.

\section{Endothelial Barrier in Inflammatory Response}

Integrity of endothelial barrier regulates the passage of materials and the transit of leukocytes in and out of bloodstream, blood clotting, angiogenesis, and vasomotor activities in microcirculation. In most organs, the endothelial cells form a dynamic barrier, keeping large plasma constituents inside of the vessel, away from tissue. In resting conditions, the vasculature leaks small solutes continuously, but restricts extravasation of large molecules and cells. In pathological process, such as inflammation, the vascular barrier is disrupted and the leakage increases. In turn, the increased extravasation leads to edema and enhances the movement of blood-borne mediators, such as activated complement components to the tissues.

Generally, increased ROS, diminished nitric oxide (NO), and impairment of signaling protein ERK5 all account for dysfunction of endothelial barrier. While the main pathways proposed for vascular leak is via endothelial junctions, formation of transendothelial 
channels from vesicles or vacuoles, as well as vesiculo-vacuolar organelles (VVO) also contribute (70). Moreover, the actin cytoskeleton may have a critical role in gap formation via the retraction of the endothelium body (71). Data from our research group also showed that human microvessel endothelial cells exposed to exogenous 2-ClHDA or 2-ClPA increased endothelium cortical stiffness, which was associated with reduced endothelial barrier function.

Taken together, the detrimental effects mediated by chlorinated lipid in addition to increased chlorinated lipid levels that have been reported under many pathologic conditions, suggest that during inflammatory process, chlorinated lipid are synthesized which in turn aggravate inflammation in a positive feedback loop. Given this correlation, it is apparent that more work is necessary to fully elucidate the effect of chlorinated lipid to elicit inflammation characterized by microcirculatory dysfunction in rat mesentery. 


\section{MATERIALS AND METHODS}

\section{Experimental Animals}

Male Sprague Dawley rats weighing 250-300g were acquired from ENVIGO (Indianapolis, IN), housed in groups of two to three with a 12:12-h light-dark cycle and allowed access to normal diet and water ad libitum. All animal protocols were approved by the Institutional Animal Care and Use Committee at the University of MissouriColumbia and were conducted in accordance with the National Institutes of Health's "Guide for the Care and Human Use of Laboratory Animals".

\section{Materials}

2-CIPA and 2-CIHDA were obtained from Center for Cardiovascular Research, Saint Louis University. Molecular probes carboxyfluorescein diacetate, succinimidyl ester (CFDA SE) was purchased from Invitrogen (Eugene, OR), and Ruthenium Red was obtained from EMD Millipore (St Charles, MO). Dihydrorhodamine 123 (DHR 123) was

purchased from Thermo Fisher Scientific (St. Louis, MO). Albumin-fluorescein isothiocyanate conjugate (FITC-albumin) and anti-MPO antibody were obtained from Sigma (St. Louis, MO) and Abcam (Cambridge, MA), respectively. Bicarbonate Buffered Saline (BBS) for exteriorized intestine and mesentery superfusion contained the following (in mM): $131.9 \mathrm{NaCl}, 4.7 \mathrm{KCl}, 2.0 \mathrm{CaCl} 2,1.2 \mathrm{MgSO} 4$, and $18 \mathrm{NaHCO}_{3}$. $\mathrm{PH}$ 7.4.

\section{Experimental Protocol}

Rats were randomly divided into four groups, 24 animals in each group. The number of animals for assessment of various parameters in each group is detailed in Table 1. After 
being anesthetized with intraperitoneal injection of a mixture of ketamine $(90 \mathrm{mg} / \mathrm{kg}$ body wt.) and xylazine (10mg/kg), a left side abdominal incision $(2-3 \mathrm{~cm}$ long $)$ was performed.

For experiments in which leukocyte/endothelial cells interactions and endothelial barrier function were assessed, the left jugular vein was cannulated to administer CFDA-SE and FITC-albumin, respectively

Table 1. The number of animals for different experimental groups.

\begin{tabular}{lllll}
\hline & ClPA & PA & ClHDA & HDA \\
\hline LA, LR and MPO activity & 6 & 6 & 6 & 6 \\
Mast cell degranulation & 6 & 6 & 6 & 6 \\
ROS and IHC staining & 6 & 6 & 6 & 6 \\
Albumin leakage & 6 & 6 & 6 & 6 \\
Total & 24 & 24 & 24 & 24 \\
\hline
\end{tabular}

The timeline for both treatment of chlorinated lipid and non-chlorinated lipid, and measurements at different time points is shown in Figure 2. At the end point, animals were sacrificed, then jejunum samples were collected for evaluation of MPO in fluorescence assay and immunohistochemistry staining.

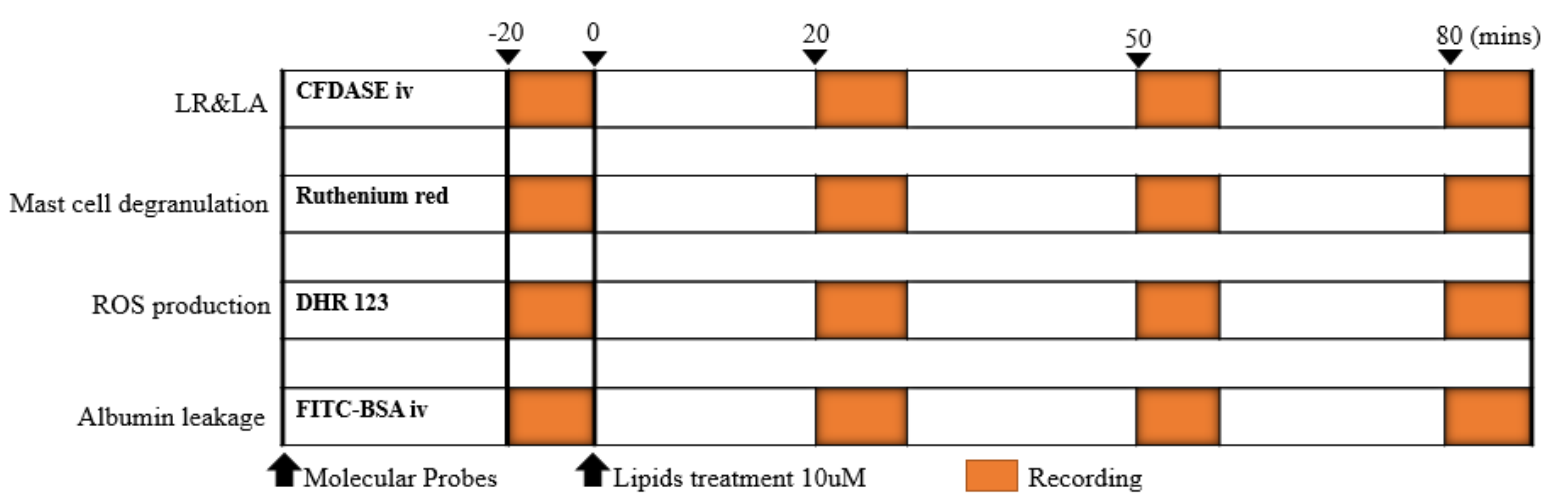

Figure 2. Timeline for different experimental groups and various parameters. 


\section{Intravital Microscopy}

As in previous studies, the rats were placed on a Plexiglas board, and the ileocecal portion of the mesentery was exteriorized and draped over a glass coverslip and superfused with a bicarbonate buffered saline $\left(37^{\circ} \mathrm{C}, \mathrm{pH} 7.4\right)$. Body temperature was maintained between $36.5-37.5^{\circ} \mathrm{C}$. The Plexiglas board was mounted on the stage of an inverted microscope (Eclips TE2000; Nikon) and the post-capillary venules in mesentery were observed, typically with 20x magnification (51). Fluorescent images (excitation, 420-490 $\mathrm{nm}$; emission $520 \mathrm{~nm}$ ) were detected with a charge-coupled device (CCD) camera (Photometrics COOLSNAP ES). Images were projected onto a television monitor (PVM1953MD; Sony) and recorded on a DVD recorder (DMR-E50; Panasonic) or captured through Metamorph software version 7.8. A time-date generator (WJ810, Panasonic) displayed this function on the monitor.

\section{Leukocyte Rolling and Adhesion}

The procedures and fluorescent labeling of leukocytes were similar to those used previously in our lab (72-74). Briefly, CFDA SE was injected through left jugular vein and the mesentery was scanned. At each time point, 10 single unbranched post-capillary venules (20-50 $\mu \mathrm{m}$ in diameter and $100 \mu \mathrm{m}$ in length) were observed for at least 30 seconds. Leukocyte/endothelial cells interactions (number of rolling and number of firmly attached) were quantified in each venule followed by calculation of the mean for the 10 venules. Leukocytes were considered to be adherent if they did not move for at least 30 seconds. Rolling cells were defined to be those passing a cross line at a velocity significantly slower than the centerline velocity and were expressed as rolling cells per minute. The numbers of adherent cells were normalized in terms of $\mathrm{mm}^{2}$ surface area. 


\section{Mast Cell Degranulation}

Ruthenium red was mixed to BBS $(0.001 \%)$ and the uptake of ruthenium red was used as a molecular marker of mast cell degranulation (75). After being exteriorized, mesentery was superfused with ruthenium red in BBS for baseline evaluation, then with ruthenium red in chlorinated lipid or non-chlorinated lipid solutions. The mesentery was scanned and for each animal, the number of degranulated mast cells in 10 fields were recorded under intravital microscope at each time point. The number of degranulated mast cell at a time point to that of the baseline was designated as the ratio of mast cell degranulation.

\section{Reactive Oxygen Species Production}

The oxidant-sensitive fluorescent probe DHR 123 was added to BBS $(10 \mu \mathrm{M})$ to estimate oxidant stress on post-capillary venular wall in rat mensentery. In brief, DHR 123 in BBS was superfused over mesentery and the mesentery was scanned for the baseline assessment, then DHR 123 in chlorinated lipid or non-chlorinated lipid solutions was applied accordingly. At each time point, 10 single unbranched post-capillary venules (20$50 \mu \mathrm{m}$ in diameter and $100 \mu \mathrm{m}$ in length) were observed and images were acquired. The fluorescence intensity was measured on the venular wall in 5 regions of interest $(25 \mu \mathrm{m}$ in diameter) (75). Since the baseline fluorescence intensity varies depending on the animal, the ratio of the fluorescence intensity at each time point to the baseline was calculated as DHR florescence ratio (51). 


\section{Albumin Leakage}

FITC-labeled albumin was used to evaluate the albumin leakage across the postcapillary venule in rat mesenteric circulation. In brief, after left jugular vein cannulation, $50 \mathrm{mg} / \mathrm{kg}$ of FITC-albumin was injected intravenously. After being exteriorized, mesentery was superfused with BBS first, and then following with chlorinated lipid or non-chlorinated lipid solutions. At each time point, 10 single unbranched post-capillary venules $(20-50 \mu \mathrm{m}$ in diameter and $100 \mu \mathrm{m}$ in length) were selected. Images were captured, and the fluorescence intensity of FITC-albumin in 5 regions of interest ( $25 \mu \mathrm{m}$ in diameter) within the venules (Iv) and in the perivenular interstitium within 10-50 $\mu \mathrm{m}$ of the venular wall (Ip) were measured using Metamorph software version 7.8. Albumin leakage was estimated by dividing Ip by Iv at each pair of corresponding circles, and the ratio of albumin leakage at a time point to that of the baseline was designated as the ratio of albumin leakage at that point (51). This method assumes that fluorescent-tagged albumin will move from the microvessel into the tissue space in a manner that reflects the behavior of native albumin.

\section{MPO activity Analysis}

Fluorescence Assay: At the end time point of experiment, rat small intestine (jejunum) was collected. Tissue MPO activity was measured in small intestine samples with use of a fluorescence assay kit (Cell Technology, Mountain View, CA). Samples were prepared as per kit manufacturer's instructions and MPO activity in the samples was quantified by adding the kit detection reagent and measuring the resulting fluorescence (excitation, $530 \mathrm{~nm}$; emission, $590 \mathrm{~nm}$ ) (76). Values were normalized to the sample protein content and MPO expression was expressed as milliuints per milligram protein. 


\section{Immunohistochemistry Staining}

The collected small intestine samples were fixed in 10\% (w/v) PBS-buffered formaldehyde and embedded in paraffin. Following dewaxing, endogenous perioxidase was quenched with 3\% (v/v) hydrogen peroxide for 5 min. The slides were incubated in $5 \%(\mathrm{v} / \mathrm{v})$ bovine serum for $20 \mathrm{~min}$ to block non-specific binding. Sections were then incubated with anti-MPO antibody (ab9535, 1:50 in PBS, Abcam) for 60 min. Samples were rinsed in wash buffer and incubated with detection system for $30 \mathrm{~min}$. Slides were applied with DAB substrate and stained with Mayer's hematoxylin for 30 seconds, then dehydrated in graded alcohol and xylene.

Immuno-histochemical images were collected using an Olympus microscope (40x) and image-pro plus software version 5.1.2.59. For graphic display of staining analysis, the immune-reactivity was expressed as the mean total number of positive staining cells. Moreover, according to the grading systems for villous injury as described, the numerical scores were the following: $0=$ normal mucosa, $1=$ development of subepithelial Gruenhagen's space and vacuolization at the villus tip, $2=$ extension of the subepithelial space with moderate lifting of epithelial layer from the lamina propria, $3=$ massive subepithelial lifting/sloughing and increased vacuolization from the tip to midportion of villi, $4=$ epithelial lifting and vacuolization from the tip to lower portion of villi, and $5=$ mucosal ulceration and disintegration of the lamina propria (77). For each group, $n=6$ and for each animal, 10 fields were randomly chosen. 


\section{Statistical Analysis}

For each animal, measurements of leukocyte rolling and adhesion, mast cell degranulation, ROS production and albumin leakage, were transformed by normalizing values in response to treatment at each time point to that animal's baseline value. Accordingly, the values presented for the aforementioned variables are the ratio of the value at each time point to the baseline. All data were shown as sample mean \pm standard error of the mean. Multiple comparisons were initially evaluated by ANOVA followed by Newman-Keuls to compare the change between control and treatment groups at three different time points. Graphpad Prism 6.0 was used to generate all statistical values. A p value of 0.05 or less is considered to be the level of statistical significance. 


\section{RESULTS}

\section{Leukocyte Rolling}

To detect the leukocyte/endothelial interactions, mesentenries exteriorized from naive rats ( $\mathrm{n}=6$ for each group) were superfused with 2-CIPA or PA, 2-CIHDA or HDA while leukocyte rolling and adhesion were measured simultaneously. The chlorinated lipid promoted leukocyte rolling, however, non-chlorinated lipid treated groups failed to exert an effect. Specifically, at $20 \mathrm{~min}, 50 \mathrm{~min}$ and $80 \mathrm{~min}$, compared to PA group $(1.7 \pm 0.68$, $1.26 \pm 0.27,1.64 \pm 0.57), 2$-CIPA group showed increased leukocyte rolling; this change became larger as superfusion continued $(4.51 \pm 1.8,5.80 \pm 1.12,6.98 \pm 2.26)$ (Figure $3 A)$. The group superfused with 2-ClHDA also showed a significant increase in leukocyte rolling that remained stable once elevated $(4.30 \pm 0.95,4.07 \pm 0.7,4.64 \pm 0.79)$, compared to HDA group at each time point $(1.54 \pm 0.76,1.47 \pm 0.41,1.96 \pm 0.75)$ (Figure $3 B)$.
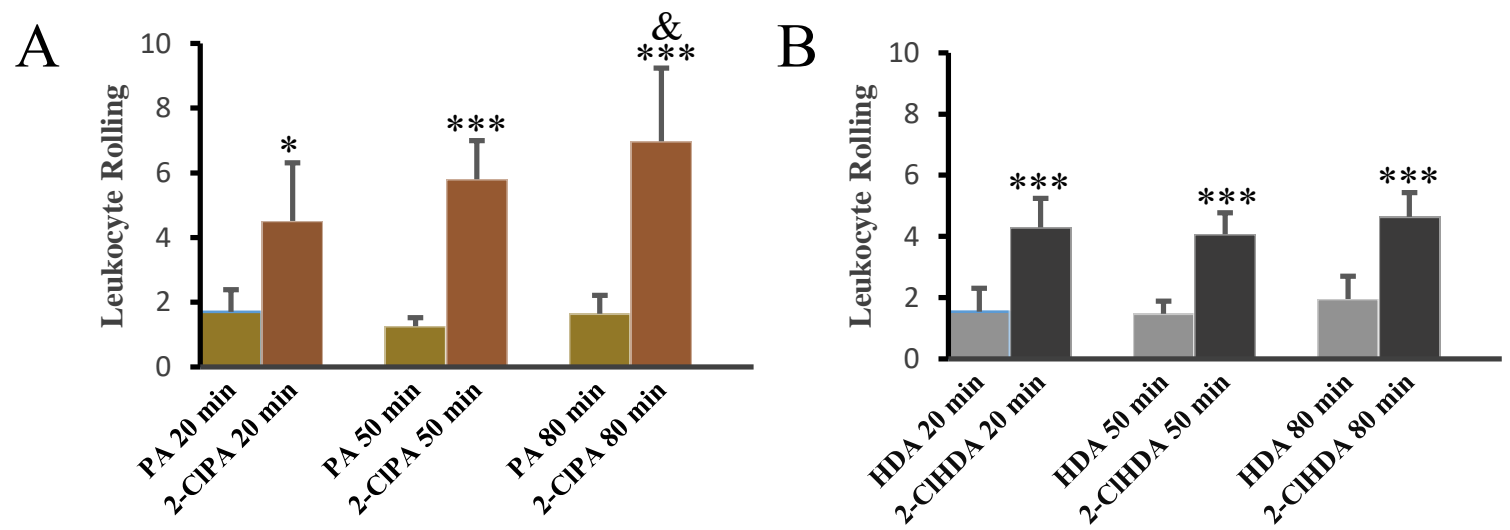

Figure 3. Chlorinated lipid promote leukocyte rolling in vivo. The number of leukocyte rolling per $\mathrm{mm}^{2}$ per minute were increased by superfusion of mensenteries with 2-CIPA and PA $(A)$, 2-ClHDA and HDA $(B)$. For each group, $\mathrm{n}=6$; and at each time point for every animal, responses in 10 postcapillary venules from intestine wall were measured. $* * * \mathrm{P}<0.001$ between groups at indicated time point, ${ }^{\circledR} \mathrm{P}<0.05$ within 2 -CIPA group, 20 min versus 80 min. 


\section{Leukocyte Adhesion}

Changes in leukocyte adhesion after treatment with chlorinated lipid or nonchlorinated lipid were also evaluated. Between 2-CIPA $(5.85 \pm 3.97,3.23 \pm 5.01,9.83 \pm 9.43)$ and PA groups $(1.07 \pm 2.61,0.84 \underline{2.06}, 6.61 \pm 7.01)$, as shown, leukocyte adhesion was increased in 2-ClPA group, which was significant only at $80 \mathrm{~min}$ (Figure 4A). Interestingly, data shows an increase trend in 2-ClHDA group, however no siginificant change was measured between 2-ClHDA $(4.73 \pm 3.08,2.96 \pm 1.76,1.56 \pm 0.7)$ and HDA groups $(2.38 \pm 2.62,2.22 \pm 2.55,5.41 \pm 2.87)$ at each time point (Figure $4 B)$.
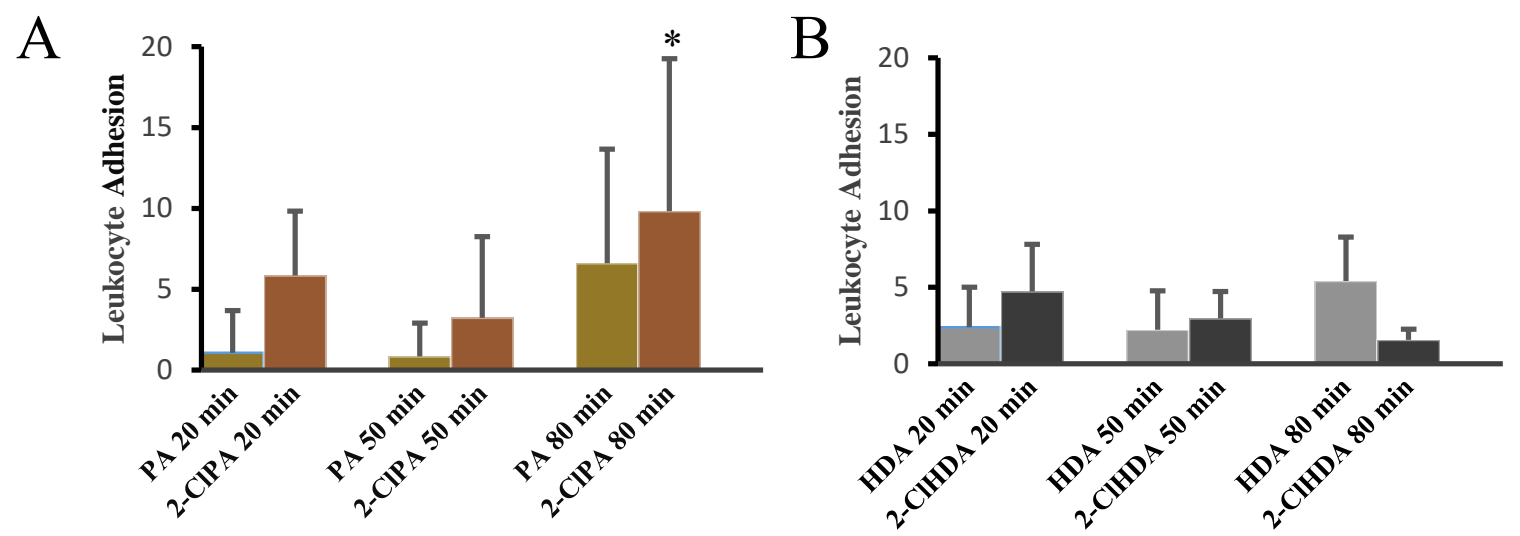

Figure 4. 2-CIPA increases leukocyte adhesion. The number of leukocyte adhesion per $\mathrm{mm}^{2}$ per 30 seconds showing leukocyte firm adhesion in response to 2-CIPA or PA (A), 2-ClHDA or HDA $(B)$ superfusion in postcapillary venule. For each group, $n=6$; and at each time point for every animal, 10 postcapillary venules from intestine wall were measured.*** $\mathrm{P}<0.001$ between 2 -ClPA and ClPA group at $80 \mathrm{~min}$. 


\section{Mast Cell Degranulation}

Mast cell degranulation was evaluated in perivascular interstitium for different groups. Representative images of non-chlorinated lipid (PA) and chlorinated lipid (2ClPA) superfusion evoked mast cell degranulation were shown respectively (Figure 5, 6). Quantitative analysis revealed that both 2-ClPA $(16.02 \pm 3.59,19.0 \pm 4.7$ and $20.37 \pm 4.83)$ and 2 -CIHDA $(8.03 \pm 0.1,8.22 \pm 0.2,7.71 \pm 0.28)$ provoked enhancement in mast cell degranulation, whereas PA exerted less effect $(3.26 \pm 0.6,3.83 \pm 0.5$ and $4.08 \pm 0.41)$ (Figure $7 A)$. Our data also showed that HDA $(3.45 \pm 0.9,4.2 \pm 1.04$ and $5.00 \pm 1.2)$ superfusion induced an increasing trend of mast cell degranulation over time. (Figure 7B).
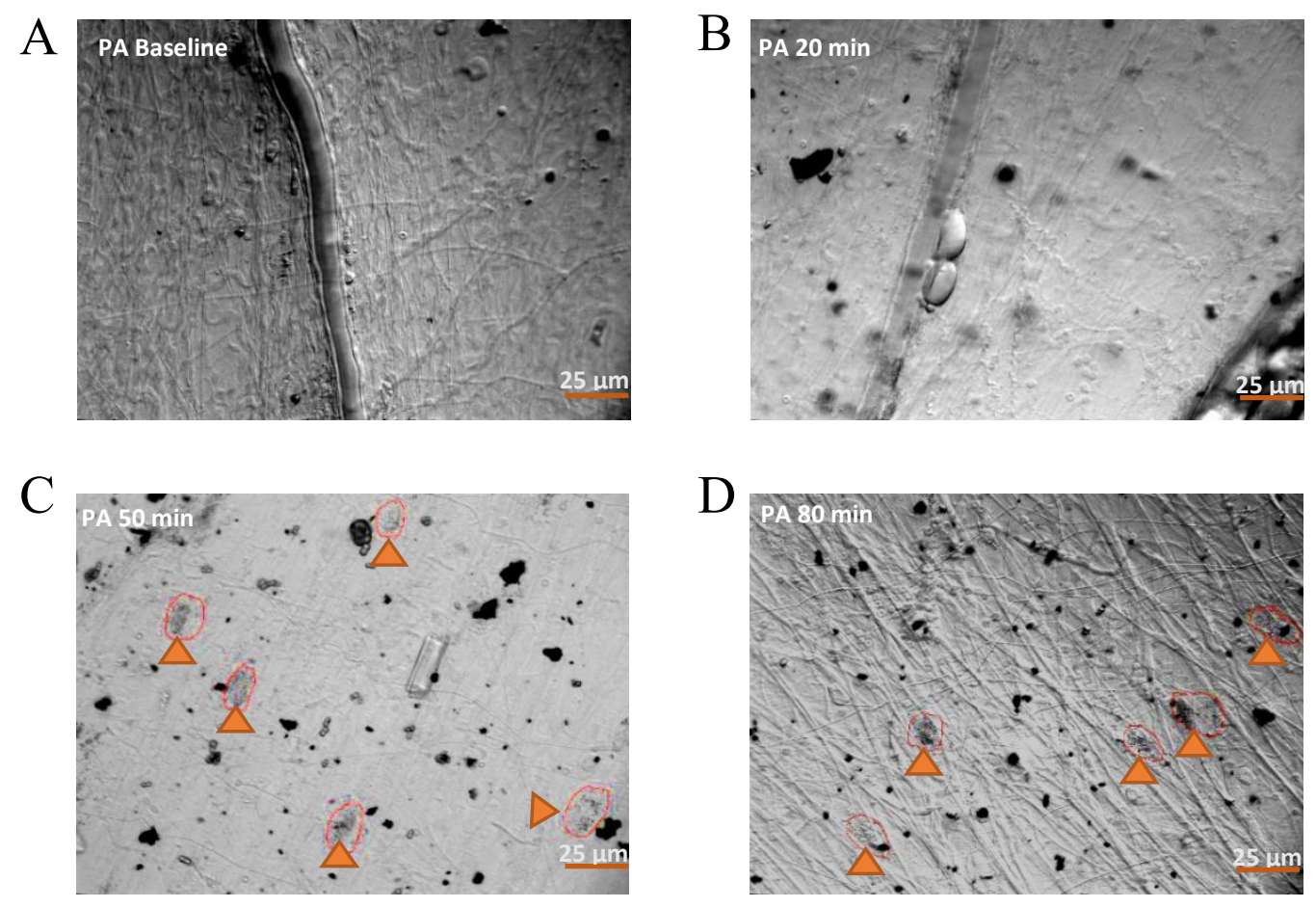

Figure 5. Representative images of mast cell degranulation responding to PA superfusion. $A$ : baseline, no degranulated mast cells were detected in rat mesentery. In contrast to 2-CIPA, PA produced very little or no mast cell degranulation after $20(B), 50(C)$ or $80(D)$ min superfusion. Yellow arrow indicates degranulated mast cells. Scale bars $=25 \mu \mathrm{m}$. 

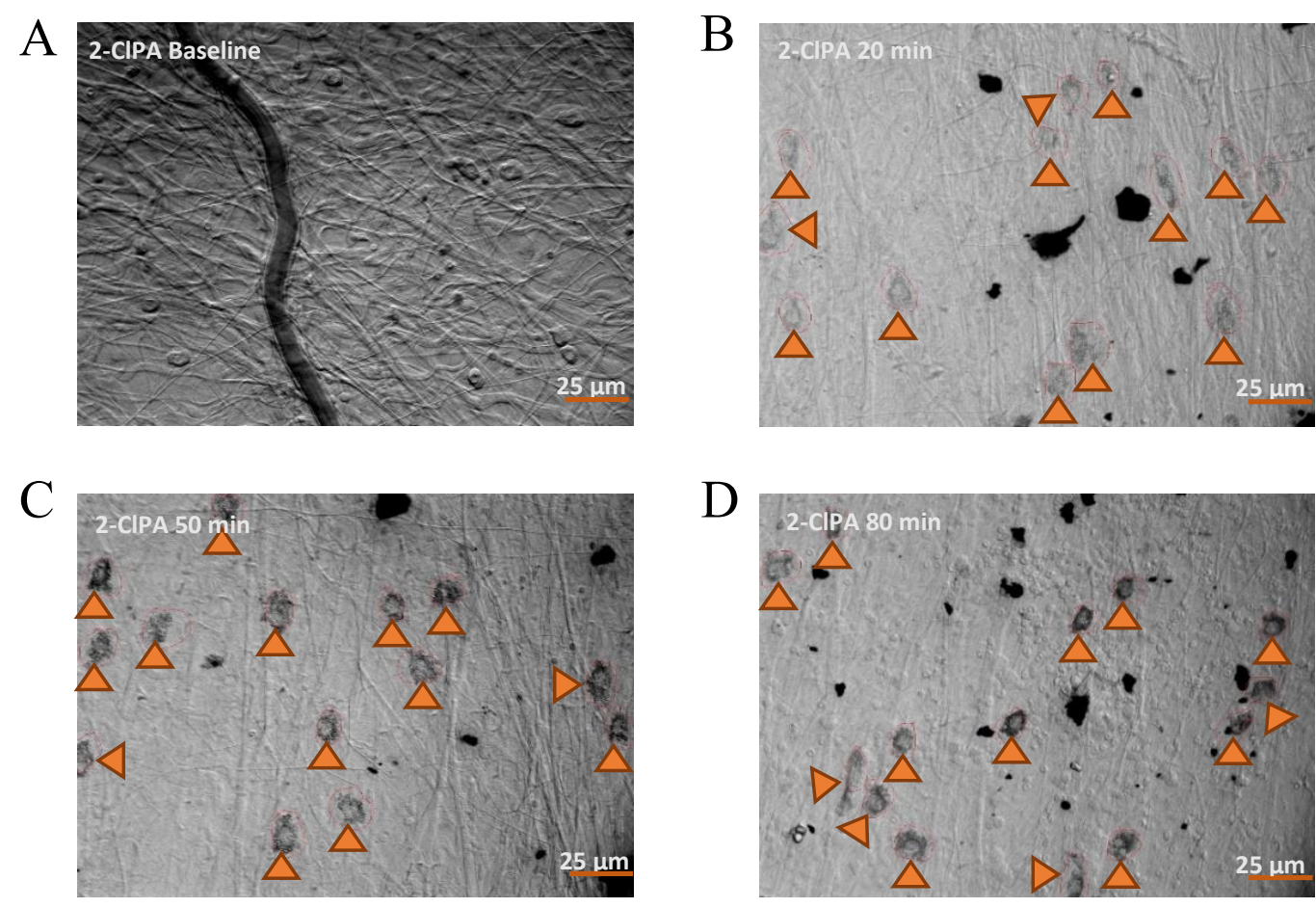

Figure 6. Representative images of mast cell degranulation responding to 2-CIPA superfusion. $A$ : baseline, no degranulated mast cells were detected in rat mesentery. $B$ : superfusion with 2-ClPA for 20 min, mast cell degranulation were detected, which increased after superfusion for $50 \mathrm{~min}(C)$ and $80 \mathrm{~min}(D)$. Yellow arrow, indicates degranulated mast cells. Scale bars $=25 \mu \mathrm{m}$.

A

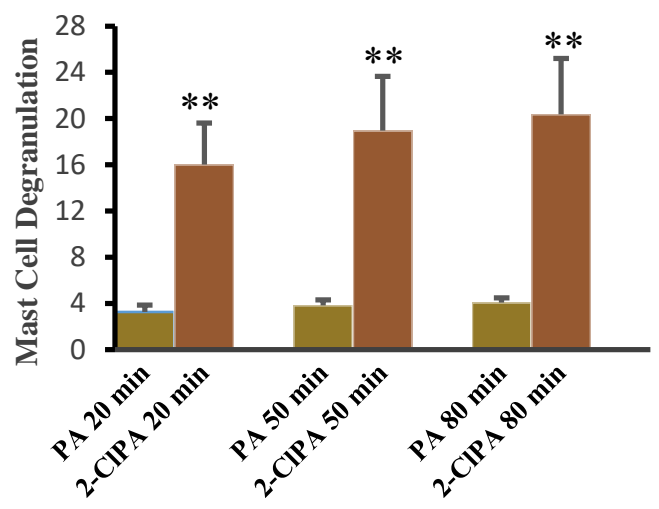

B

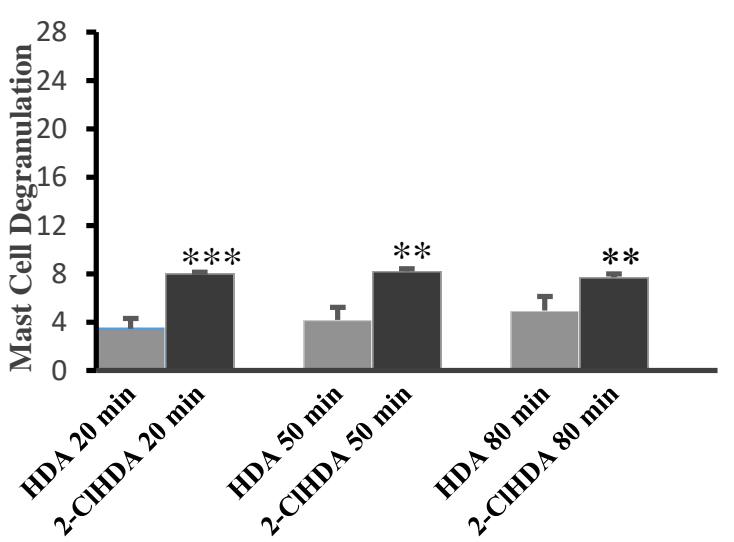

Figure 7. Mast cell degranulation changes with chlorinated lipid superfusion. The number of degranulated mast cell per field, in response to 2-CIPA or PA $(A)$, 2-ClHDA or HDA $(B)$ superfusion in peripostcapillary venules were counted. Respective mean value at different time point was normalized to the baseline, shown as sham group here. For each group, $n=6$; and at each time point for every animal, 10 fields (20x) along mesenteric venules were measured. ${ }^{* * *} \mathrm{P}<0.001, * * \mathrm{P}<0.01$ between groups at indicated time point. 


\section{ROS Production}

When fluoresence intensity of DHR 123 in the venular walls was initially measured, no or very little DHR fluoresence was detected on rat mesenteric venular walls at baseline. Chlorinated lipid induced a pronounced DHR fluoresence on mesenteric venular walls, and the representative images of 2-ClHDA group are illustrated in Figure $8(A-D)$.
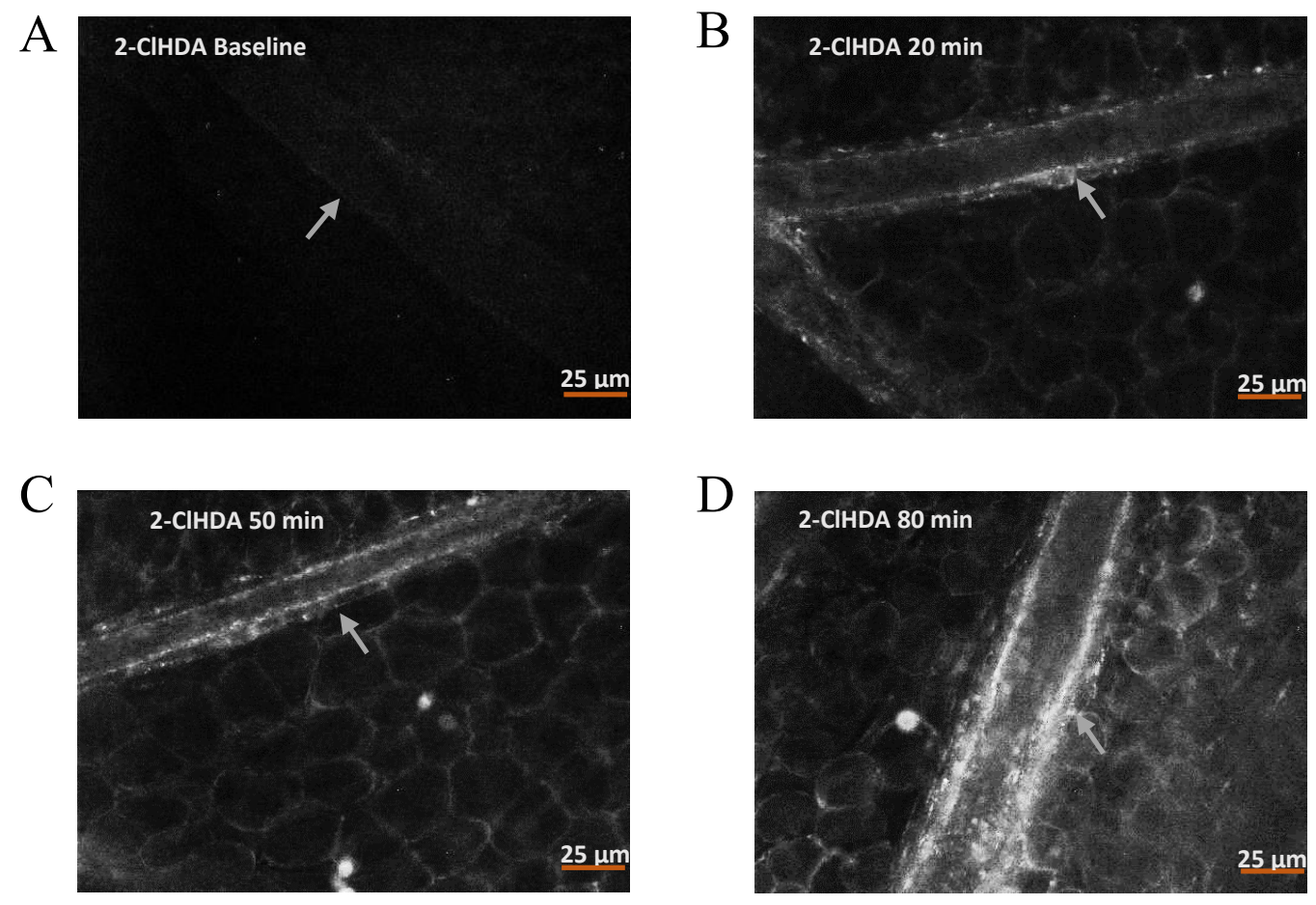

Figure 8. Representative images of ROS production on mesenteric venular wall. $A$ : baseline, no DHR fluoresence is visible. $B$ : 20 min after 2-ClHDA superfusion, detectable DHR fluoresence shows on the rat mesenteric venule wall. $C$ : $50 \mathrm{~min}$ after 2-ClHDA superfusion, more fluorescence is showing on venular wall. $D: 80 \mathrm{~min}$ after superfusion, the fluoresence are much brighter. White arrow indicates mesenteric venule. Scale bars $=25 \mu \mathrm{m}$. 
Specifically, with 2-CIPA treatment, DHR fluoresence was elevated throughout the superfusion with this chlorinated lipid $(2.48 \pm 0.91,2.28 \pm 0.42$ and $2.41 \pm 0.33)$ (Figure 9A). Moreover, the fluoresence intensity after 2-ClHDA treatment was also increased over time $(1.71 \pm 0.14,2.08 \pm 0.13,2.59 \pm 0.26)$, being especially prominent at $80 \mathrm{~min}$ when compared to that at 20 min (Figure 9B). On the contrary, in the non-chlorinated lipid groups, there was no significant changes in the DHR fluoresence ratio on the venular wall throughout the observation. Specifically, the fluoresence ratio was $1.20 \pm 0.26,1.51 \pm 0.48$ and $1.34 \pm 0.28$ in PA group, and $1.07 \pm 0.13,1.23 \pm 0.1$ and $1.33 \pm 0.16$ in HDA group at $20 \mathrm{~min}$, $50 \mathrm{~min}$ and $80 \mathrm{~min}$ respectively.
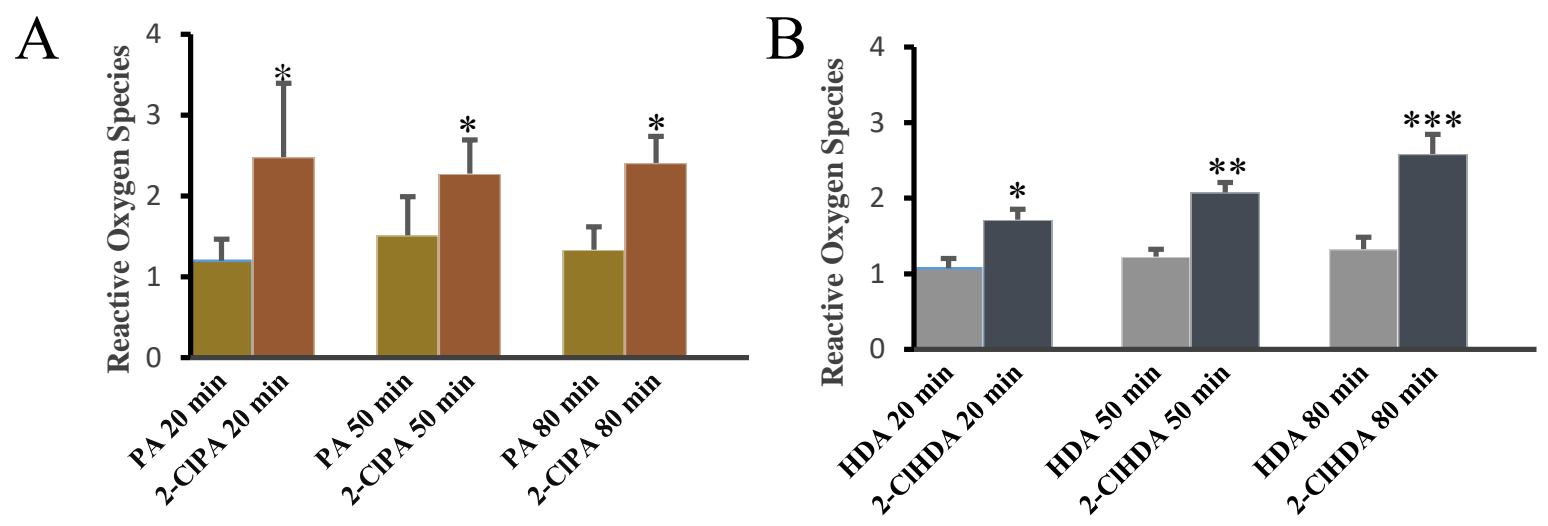

Figure 9. Chlorinated lipid increase ROS production in mesentery. The ratio of DHR 123 fluorescence intensity along the vesule wall at each time point to that at baseline indicated reactive oxygen species production responding to 2-CIPA or PA (A), 2-ClHDA or HDA $(B)$ superfusion in mesentery. For each group $(n=6)$, at each time point for every animal, 10 mesenteric venules were randomly chosen and 5 regions of interest ( $25 \mathrm{um}$ in diameter) along venule wall were measured. $* * * \mathrm{P}<0.001, * * \mathrm{P}<0.01, * \mathrm{P}<0.05$ between groups at indicated time point, ${ }^{\&} \mathrm{P}<0.05$ within 2 -ClHDA group, $20 \mathrm{~min}$ versus $80 \mathrm{~min}$. 


\section{Albumin Leakage}

The representative images of FITC-labeled albumin leakage from rat mesentery venules challenged with chlorinated lipid are presented (Figure 10). No or very limited albumin leakage was observed at baseline, whereas leakage was apparent when observed at $20 \mathrm{~min}, 50 \mathrm{~min}$ and $80 \mathrm{~min}$ with chlorinated lipid superfusion (Figure $10 A-D$ ).
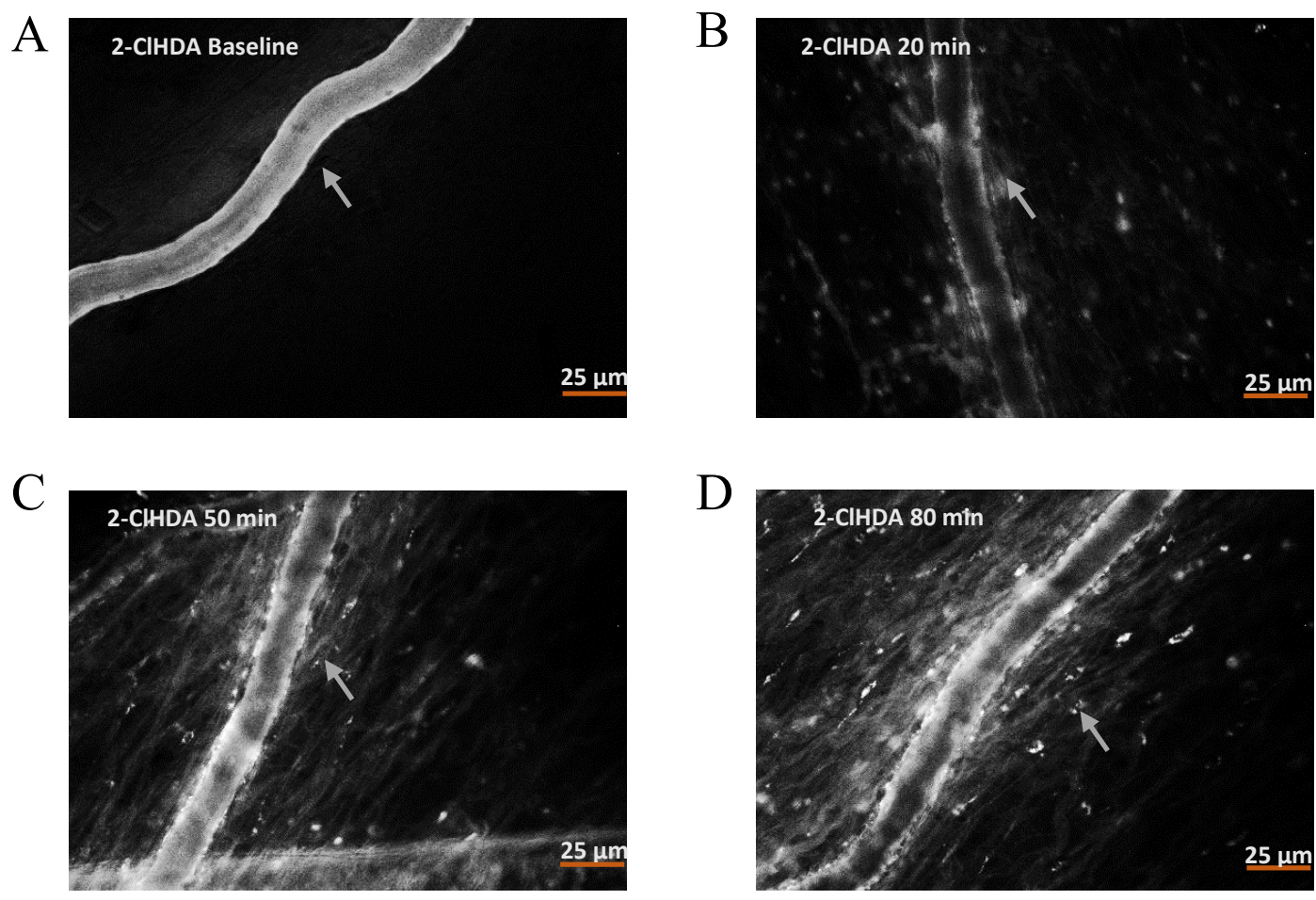

Figure 10. FITC-labeled albumin leakage from rat mesentery venules. $A$ : baseline, all FITC-labeled albumin is localized within venule, and no labeled albumin is visible in the perivenular interstitium. $B$ : superfused with 2-ClHDA for $20 \mathrm{~min}$, detectable fluoresence shows in the perivenular interstitium (Ip) while decrease fluoresence within venules (Iv). C: $50 \mathrm{~min}$ with 2-ClHDA superfusion, with an increase in Ip, fluorescence intensity decreases in Iv. $D$ : superfusion for $80 \mathrm{~min}$, the comparison between Ip and Iv is more obvious. White arrow indicates postcapillary venules in rat mesentery. Scale bars $=25 \mu \mathrm{m}$. 
The albumin leakage from the rat mesenteric venules was quantitated and graphed against time (Figure 11). The albumin leakage was very low or even undetectable before lipid treatment in all groups, and this situation persisted throughout the observation period in mesenteric superfused with PA $(1.11 \pm 0.21,1.08 \pm 0.16$ and $1.03 \pm 0.04)$ or HDA $(0.98 \pm 0.05,0.97 \pm 0.15$ and $1.07 \pm 0.17)$. On the other hand, in the 2-ClPA $(3.19 \pm 0.003$, $3.56 \pm 0.19$ and $4.0 \pm 0.17)$ and $2-\operatorname{ClHDA}(2.13 \pm 0.49,2.7 \pm 0.53,3.21 \pm 0.6)$ group, albumin leakage from venules increased immediately after the initiation of chlorinated lipid superfusion in a time-dependent manner.
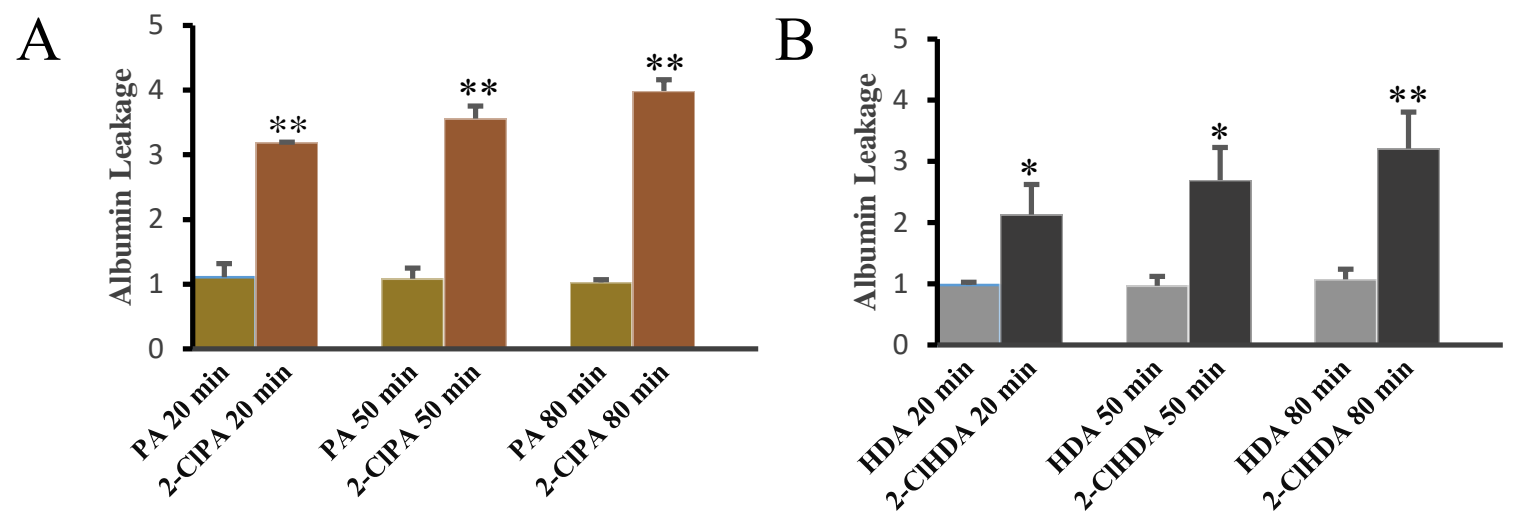

Figure 11. FITC-albumin leakage in mesenteric microcirculation along with chlorinated lipid treatment. The fluoresence intensity within mesenteric postcapillary venules (Iv) and in perivenular interstitium (Ip) in response to 2-CIPA or PA $(A)$, 2-CIHDA or HDA $(B)$ superfusion were measured. For each group $(\mathrm{n}=6)$, at each time point for every animal, 10 mesenteric venules were randomly chosen and 5 regions of interest $(\mathrm{r}=12.5 \mathrm{~mm})$ along venule wall were measured. The ratio of $\mathrm{Ip} / \mathrm{Iv}$ was calculated at each coresponding cirle, and mean value of the ratio at each time point was normalized to baseline. ${ }^{*} * \mathrm{P}<0.01$, $* \mathrm{P}<0.05$ between groups at indicated time point. 


\section{MPO Activity Assay}

In addition to the aforementioned indices of inflammation which were shown to increase after chlorinated lipid exposure, we also assessed MPO levels as a marker of neutrophil infiltration. Rat small intestine, when subjected to chlorinated lipid superfusion till the end of experiment, contained more abundant brown staining compared to nonchlorinated lipid groups, as determined by immunohistochemistry staining and revealed by high magnification (40x). Of note, when compared 2-CIPA to PA, or 2-CIHDA to HDA, in submucosa of small intestine more MPO was detected in chlorinated lipid groups (Figure 12, A-D). However, based on the staining, no significant injuries was observed in epithelium of small intestine in four groups (data were not shown here).

A

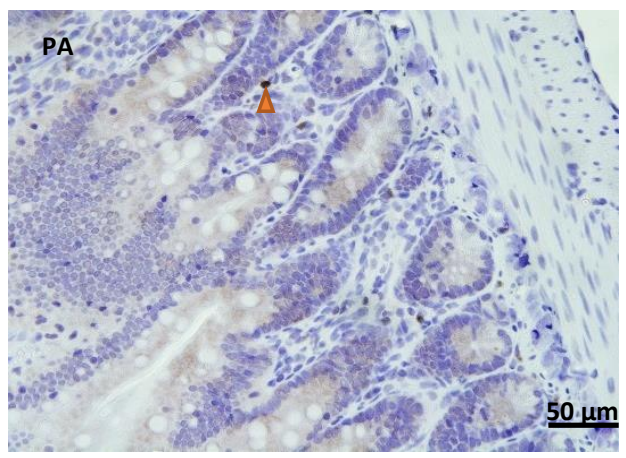

$\mathrm{C}$

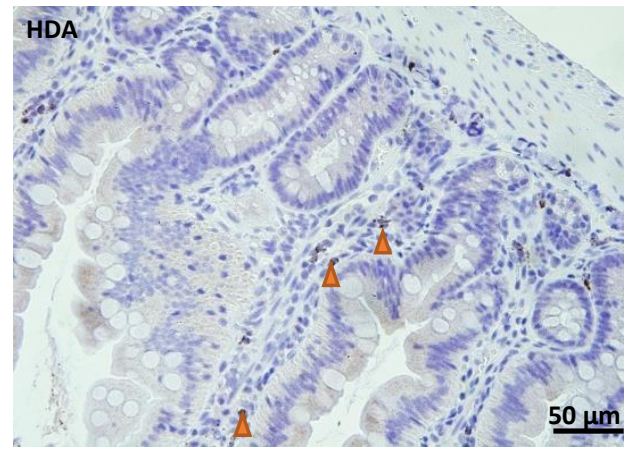

$\mathrm{B}$

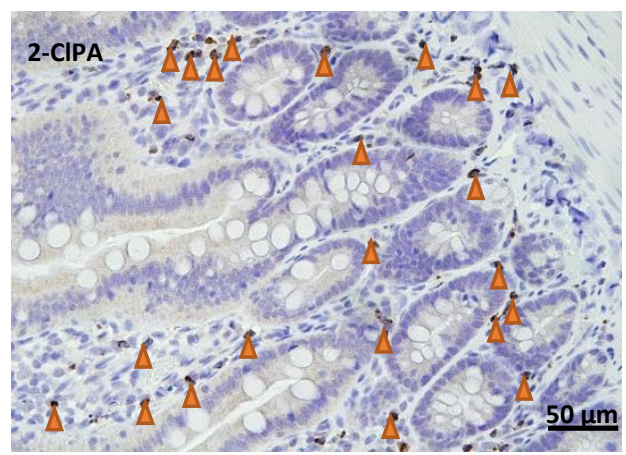

$\mathrm{D}$

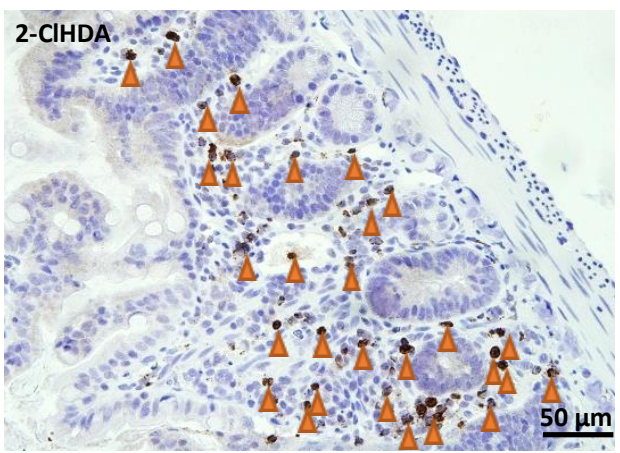

Figure 12. IHC staining for MPO in small intestine. Immunohistochemistry staining of myeloperoxidase in rat small intestine, as shown in brown staining. A: 2-CIPA; $B$ : PA; $C$ : 2-ClHDA; $D$ : HDA. Yellow arrow head indicates positive staining (brown) cells. Scale bars $=50 \mu \mathrm{m}$. 
Quantitative analysis of IHC staining for MPO in submucosa of rat small intestine exposed to 2-CIPA $(37.07 \pm 5.46)$ or $2-\mathrm{ClHDA}(40.28 \pm 4.75)$ induced increased positive staining cells in relative to control groups as shown (PA: 12.53 \pm 1.6 ; HDA: 12.98 \pm 1.25 ) (Figure 13A). Given the markedly increased MPO staining in rat mesentery superfused with chlorinated lipid, we explored the putative effect of chlorinated lipid on MPO activity in the mucosa. Similar to our IHC results, MPO levels were significantly increased in chlorinated lipid treated animals (2-ClPA: 1434.46+243.82; 2-ClHDA: 1568.4+409.18) compared with the non-chlorinated controls (PA: 818.86 \pm 95.97 ; HDA 720.24 \pm 102.31 ) (Figure 13B).
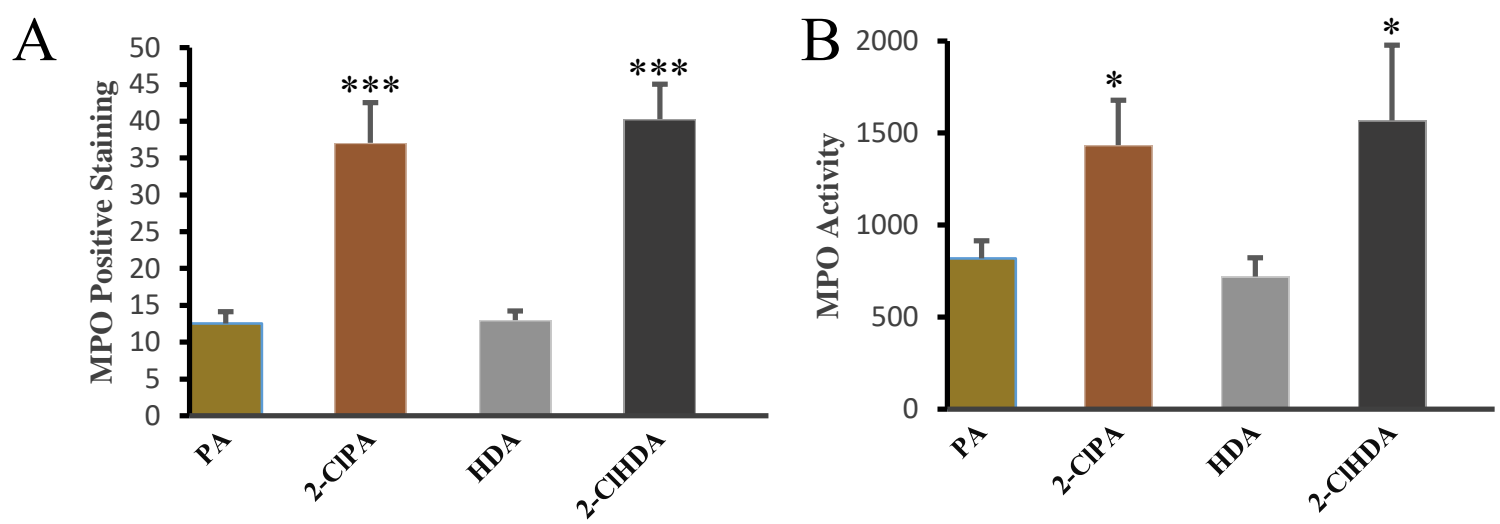

Figure 13. MPO activity after chlorinated lipid challenge. A: Quantification of IHC positive staining cells for MPO in small intestine collected at the end time point. For each group $(n=6), 10$ fields under microscpoe (40x) focused on submucosa were randomly chosen. $B$ : Tissue MPO activity was analyzed by measuring resulting fluoresence at $530 \mathrm{~nm}$ (exitation) and $590 \mathrm{~nm}$ (emission). The measurements were reapeated for three times and mean values were normalized for sample protein content $(\mathrm{n}=6) . * * * \mathrm{P}<0.001, * \mathrm{P}<0.05$ between groups at indicated time point. 


\section{DISCUSSION}

The primary purpose of this study was to test the hypothesis that, when compared to mesenteric superfusion with non-chlorinated lipid, rat mesenteries will exhibit endothelial and microcirculatory dysfunction in response to chlorinated lipid. Impaired microcirculatory function with increased plasma 2-chlorofatty acid (2-ClFA) level is well established in septic patients and the rat cecal ligation and puncture model of sepsis. However, the link between elevated chlorinated lipid levels and development of microcirculatory dysfunction has not been established. Moreover, the mechanisms underlying such sepsis-induced changes in vivo are multi-factorial and complex due to integration of several proinflammatory factors and the involvement of multiple organs. For instance, microbial invasion in sepsis elicits powerful immune responses that involve release of pro-inflammatory mediators, over-exuberant production of reactive oxygen species (ROS), mast cell degranulation, leukocyte-endothelial cell interactions, and microvascular barrier disruption that serve as a motor for multi-organ failure when these responses extend systemically. Microcirculatory function is severely compromised in sepsis, which further aggravates inflammation. Neutrophil/endothelial cell interactions, characterized by increased leukocyte rolling and adhesion along the lumen of post capillary venules followed by emigration into the tissues where they direct a focused attack on parenchymal cells, is a major contributor to cell dysfunction. Given these considerations, we wished to determine the local effects of exposure to chlorinated lipid in the absence of confounding compromise in the function of multiple organs, as occurs in sepsis.

One of the hallmark feature of inflammation is endothelial barrier dysfunction, or disrupted endothelial barrier in microcirculation. This is especially problematic for the 
lungs, where edema formation and alveolar flooding impairs gas exchange. Chlorinated lipid associated inflammatory response may cause alterations in microcirculatory functions; however, it is difficult to rigidly isolate all systemic variables in vivo. The working model in naïve rats with local exposure of only a small region of mesentery allows independent, precise superfusion (shown in Table 1) under experimentally controlled conditions, enabling direct treatment of chlorinated lipid, 2-ClPA and 2-ClHDA, or nonchlorinated lipid, PA and HDA as controls, as a result, and examination of inflammatory responses. This avoids the complications associated with impaired gas exchange and resulting hypoxia in the production of inflammatory responses secondary to production of chlorinated lipid. This study quantified the inflammatory response of rat mesentery to chlorinated and non-chlorinated lipid superfusion via intravital microscopy.

In addition, with chlorinated lipid superfusion, endothelial barrier dysfunction was observed in microvessel endothelial cells while increased cell surface expression of Pselectin, E-selectin, VCAM-1 and ICAM-1 in cells treated with 2-CIHDA and 2-CIPA in human endothelial cell. While in vitro measurement allows monitoring of immediate effect on endothelial dysfunction and endothelial barrier disruption induced by chlorinated lipid, it is more difficult to predict the relationship between chlorinated lipid and inflammation due to the complex environment in the body, wherein several cell types, mediators release from the extracellular matrix, and parenchymal cell injury all contribute to systemic inflammation. Therefore, we sought to directly measure the pro-inflammatory effects of 2CIPA or 2-ClHDA on the intact microcirculation in vivo to measure neutrophil/endothelial cell interactions, ROS production, and mast cell degranulation and albumin leakage directly with controlled superfusion in vivo. 
As predictive, we uncovered two distinct responses in rat mesentery. The first consisted of rats which responded to 2-ClPA and 2-ClHDA respectively with an increase in leukocyte-endothelium interactions and a second group, which responded to PA and HDA respectively without an increase in leukocyte-endothelium interactions. These experiments showed profound differences in leukocyte rolling and adhesion with different superfusion challenges. E-selectin and P-selectin expressed on activated endothelial cells could interact with L-selectin and P-selectin glycoprotein ligand 1 (PSGL-1) on neutrophils to mediate leukocyte rolling and adhesion (51). Previous studies by McHowat et al have shown that human endothelial cells treated with 2-ClPA or 2-ClHDA had significantly increased surface expression of P-selectin, E-selectin, VCAM-1 and ICAM-1. In addition, CD62L and CD66b are adhesion molecules expressed on activated neutrophils, allowing their adherence to endothelial cells. Moreover, binding of integrin $\mathrm{CD} 11 \mathrm{a} / \mathrm{CD} 18$ on activated leukocytes surface with responding receptor ICAM-1 on endothelial cells, works to strengthen neutrophil/endothelial cell interactions (51). Although in vitro measurements including isolated postcapillary venules and cell culture models incorporating shear stress exposure (78-80), have the advantage of assessing individual contribution to leukocyteendothelial cells interactions, in vivo working mesentery preparations give the advantage of describing microcirculation function within the organism and allowing investigation the effect of chlorinated lipid with associated neuronal and humoral modulation of microcirculation. Increased leukocyte rolling and adhesion occurred in response to mesenteric superfusion with chlorinated lipid.

Similarly, both 2-ClPA and 2-ClHDA superfusion promoted mast cell degranulation when compared to the non-chlorinated lipid treatment groups. It has been shown by others 
that chloride and hypochlorite products from $\mathrm{MPO}-\mathrm{H}_{2} \mathrm{O}_{2}$-chloride system promote neutrophil extracellular trap (NETs) formation, which is responsible for immobilizing and killing invading microbes (59). Activated neutrophils could also secrete oxygen radicals as well as proteolytic enzymes, including serine protease proteinase-3 (PR-3), elastase and cathepsin G, leading to endothelial cell injury (81). Moreover, 2-ClHDA could mediate microvascular endothelial cell dysfunction, such as loss of barrier function, mitochondrial dysfunction, and apoptosis (34). Of note, based on the likely interaction between mast cells and neutrophils, the granular content of mast cells such as proinflammatory mediators and cytokines, could be released through a "kiss and run" mechanism (82). Kiss-and-run fusion is a type of vesicle release or exocytosis where the vesicle docks and transiently fuses at the plasma membrane and releases its granule contents, after which the vesicle can then be reused. The process of mast cell degranulation can be mediated by both immune and non-immune pathways. The immune pathway is usually activated by aggregation of specific surface receptors FceRI binding with several complexes of antigens with IgE. The $\gamma$-chains of the receptor mediating signal transmission, leads to an increase in $\mathrm{Ca}^{2+}$ concentration in the cytosol, which initiates degranulation and synthesis of new mediators. On the contrary, non-immune pathways are triggered by cytokines, chemokines, components of cell wall and some other substances $(61 ; 83)$. Secreted tryptases and chymases from mast cells could promote inflammation, matrix destruction and tissue remodeling via destroying pro-coagulant, activating proteinase-activated receptors, and promoting the conversion of angiotensin I to angiotensin II (63).

In addition to enhanced leukocyte-endothelial adhesive interactions and mast cell degranulation, we also noted reactive oxidative species (ROS) production in postcapillary 
venules on mesenteric exposure to 2-CIPA and 2-CIHDA challenges, respectively. Based on these results, we postulated that chlorinated lipid are associated with ROS production in vivo. The mechanisms underlying this ROS production could fall into one of several categories. 2-ClHDA has been shown to be a potent neutrophil chemoattractant, inhibitor of endothelial nitric oxide synthase (eNOS), inducer of the expression of cyclooxygenase2 (COX-2) and mediator of endothelial cell dysfunction (84-87). By these mechanisms, activated neutrophils could potentially play a role in the observed changes in ROS production. Activated neutrophils consume oxygen to generate an array of damaging reactive oxidizing species such as superoxide anions and hydrogen peroxide $(10 ; 88)$. As previously mentioned, hydrogen peroxide is released concomitant with the release of heme protein MPO for production of hypochlorous acid $(\mathrm{HOCl})$. A further explanation of ROS production could also include endoplasmic reticulum (ER) stress. It should be noted that 2-ClHDA can be oxidized intracellularly to 2-ClPA, which solely can lead to $\mathrm{H}_{2} \mathrm{O}_{2}$ production from THP-1 monocytes (80). The application of the antioxidant, Nacetylcysteine (NAC) and glutathione (GSH) alleviated release of $\mathrm{H}_{2} \mathrm{O}_{2}$ induced by 2-ClPA (80), and attenuated 2-CIPA induced caspase 3 activity, poly ADP-ribose polymerase (PARP) cleavage and CCAAT/enhancer-binding protein homologous protein (CHOP). The interplay between ROS and ER stress has been reported in many studies, while the detailed mechanism is still not well understood $(89 ; 90)$. Investigation of $\alpha$-tocopheryl succinate-treated human gastric carcinoma cells implied that ROS generation decrease results from attenuation of ER stress, and the major pathways involved are inositol requiring enzyme-1 (IRE1) and RNA-dependent protein kinase-like ER kinase (PERK) (91). In addition, the effect of chlorinated lipid to induce mast cell degranulation may result 
in chymase release. This enzyme catalyzes the conversion of angiotensin I to angiotensin II, a known activator of NADPH oxidase.

Our work also shows that chlorinated lipid also increased vascular permeability. Here the data showed that in the 2-CIPA and 2-ClHDA group, albumin leakage from venules increased immediately after the initiation of chlorinated lipid superfusion in a timedependent manner. In general, increased ROS, diminished nitric oxide (NO), and impairment of signaling protein ERK5 may all contribute to dysfunction of endothelial barrier. The main pathways for vascular leak of protein like albumin, include endothelial junctions, vesicular shuttling, and/or formation of transendothelial channels from vesicles or vacuoles into vesiculo-vacuolar organelles (VVO) (70). The paracellular junctions lie between endothelial cells are organized into adherens and tight junctions, and the main component of adheren junctions is vascular endothelial (VE)-cadherin, forming homophilic complexes (92). Adherens junctions dissolve in response to inflammatory cytokines such as histamine and bradykinin, allowing extravasation of macromolecules. The mechanisms under this involves VE-cadherin dissolution, which may result from direct or indirect phosphorylation mediated by activation of c-Src (93). However, the mechanism of VE-cadherin internalization remains to be identified (94). In addition, the VVO is a distinct endothelial cell structure that provides a transcellular pathway for macromolecular extravasation in endothelial transcytosis under particular conditions (9597). Vesicles and vacuoles that make up VVO were originally thought to derive from caveolae, with caveolin-1 as the main protein. However, the fact that caveolin-1 knock-out mice still exhibit VVOs in vasculature, implies caveolin-1 may not be required for VVO formation. 
In addition to these mechanisms, the actin cytoskeleton may have a critical role in gap formation via the retraction of the endothelium body (71). Our laboratory has also showed that human microvessel endothelial cells exposed to exogenous 2-ClHDA or 2ClPA treatment showed increased endothelium cortical stiffness, which coincided with reduced endothelial barrier function. The mechanisms could be action of intracellular motor proteins facilitates opening of paracellular junctions. It is also possible that the cell shape changes could be a natural recoil occurring during paracellular junctions disassembly (98; 99). Small guanosine triphosphatases (GTPase) from the ras superfamily, primarily Rho GTPases (RhoA, Rac1, Cdc42) or Rap1, affect microvascular permeability via reorganization of junction-associated cortical actin cytoskeleton. Specifically, Rac1 and Cdc42 are the main GTPases required for barrier maintenance and stabilization, while RhoA negatively regulates barrier functions (100-103). It is also known that cAMP signaling enhances barrier functions partially via Rap1 mediated activation of Rac1 and Cdc42 as well as inhibition of RhoA (104). Inhibition of these signaling pathways by chlorinated lipid may also underlie the increased permeability. Clearly, much additional work will have to be undertaken to address these intriguing hypotheses.

We hypothesized that compared to non-chlorinated lipid, chlorinated lipid could elicit inflammation via a positive feedback cycle via the activation of neutrophil-MPO system. Based on our IHC staining and MPO fluorescence assay, MPO level was significantly increased in chlorinated lipid treated animals compared with the non-chlorinated controls. This key enzyme, primarily derived from activated neutrophils, induces endothelial dysfunction and caspase activation resulting in human endothelial cells apoptosis via oxidative stress (105-107). It has been suggested that MPO in neutrophils diminishes the 
toxic effects and mortality in sepsis model induced by lipopolysaccharide (108). The underlying mechanisms could be that MPO binds to and activates neutrophils, supports cell adhesion, and prolongs neutrophil survival via interactions with CD11b/CD18 integrin and electrostatic interactions (109-112). MPO binds to red blood cell membranes, and is transported by red blood cells to remote sites, affecting endothelial function as well as systemic vascular resistance (113). However, MPO can also have anti-inflammatory effects directly by its catalytic action, via the oxidative modification or destruction of soluble mediators, such as high-mobility group protein 1 (HMGB1) $(114 ; 115)$. Based on this, further study will test whether preventing MPO-induced chlorinated lipid production could reduce inflammatory injury in vivo.

In the current studies, we showed that 2-CIPA and 2-CIHDA caused similar endothelial responses and ROS production in the rat mesenteric microcirculation, while 2CIPA induced more mast cell degranulation at all the time points and albumin leakage at $20 \mathrm{~min}$ and $50 \mathrm{~min}$. Other studies have demonstrated that neutrophils and endothelial cells can metabolize 2-ClHDA (an $\alpha$-chlorofatty aldehyde) to 2-ClPA (an $\alpha$-chlorofatty acid) and 2-ClFOH (an $\alpha$-chlorofatty alcohol) (4). Reaction of $\alpha$-chlorofatty aldehydes with the nucleophile glutathione (GSH) in activated human neutrophils and in vivo has been shown to promote the formation of glutathione adducts (116). On the other hand, $\alpha$-chlorofatty aldehydes can also elicit functional changes in targeted cells though Schiff-base adduct formation with primary amines of proteins and lipids (117). In this regard, 2-ClHDA Schiff-base adducts with ethanolamine glycerol-phospholipids and lysine have been identified (118). Although 2-CIHDA and 2-CIPA can act as a chemoattractant and a chemorepellant, respectively, for neutrophils, both of 2-ClHDA and 2-ClPA increase 
endothelial COX-2 levels (32) (18). Since 2-ClHDA can be converted to 2-CIPA, it is possible that the effect of 2-ClHDA exposure to increase NF- $\kappa \mathrm{B}$ dependent $\mathrm{COX}-2$ expression may be mediated by this secondarily derived metabolite, rather than 2-ClHDA per se $(32 ; 33)$. This is a possible explanation for higher mast cell degranulation and albumin leakage induced by 2-ClPA when compared with 2-ClHDA. However, the relative contributions of 2-ClHDA versus its metabolites on these responses remain to be investigated.

Local superfusion with chlorinated lipid allows examination of local inflammatory responses that are not likely complicated by systemic effects (however, see below). Nevertheless, several limitations of the working model are notable. The mesentery was the site of exposure to chlorinated lipid. Therefore, the inflammatory responses we observed represent local inflammatory responses that are not influenced by effects of systemic exposure to chlorinated lipid that characterizes sepsis. For one thing, superfusion of the rat mesentery with compounds is a well-established delivery system, which implies that chlorinated lipid superfused could be absorbed rapidly via mesenteric microcirculation causing systemic inflammation. However, the mesenteric surface area is small in our model. For another, Paul et al indicated that following a sterile injury to visceral organs, a reservoir of fully mature peritoneal cavity macrophages can respond within an hour (119). In this case, we could explain our results as chlorinated lipid activate macrophages or neutrophils, leading to the increase of MPO and other inflammatory responses that extend beyond local exposure. Other drawbacks of using the exteriorized mesentery superfusion method include vulnerability to injury during exteriorizing procedures and throughout the protocol, increased reactive oxidative stress and progressive loss of mesentery function 
over time as a result of tissue exposure. As noted above, the inflammatory milieu is characterized by a larger number of factors extrinsic to the postcapillary venular walls that influence neutrophil/endothelial cell interactions, venular permeability responses and so on. It is necessary to perfect our approaches that allow precise control of these external factors, making the individual contributions to mesenteric microcirculation dysfunction more reliable.

The result of our study represents the first in vivo characterization of inflammatory responses. Further experiments elucidating the mechanisms underlying these responses and examining other pro- and anti- inflammatory mediators are required to explain how these microcirculatory alterations occur. Given the observations of increased P-slectin, Eselecin, ICAM-1 and VCAM-1 in human endothelial cells with 2-CIPA and 2-CIHDA treatment, subsequent experimentation will consist of western blotting in tissue samples from animals to quantify adhesion molecules expression change with 2-CIPA and 2ClHDA superfusion in vivo, respectively. Given the possibility of chlorinated lipidneutrophil activation-MPO-chlorinated lipid positive feedback loop, further experimentation could also be performed to specifically block myeloperoxidase (MPO) oxidation in rat sepsis model with $\mathrm{N}$-acetyl lysyltyrosylcysteine amide (KYC) (Figure 14). Under inflammation ablation in septic rats would explain that in sepsis, chlorinated lipid elicit inflammation via a positive feedback way to activate neutrophils entering into MPOHOCl-chlorinated lipid loop. 


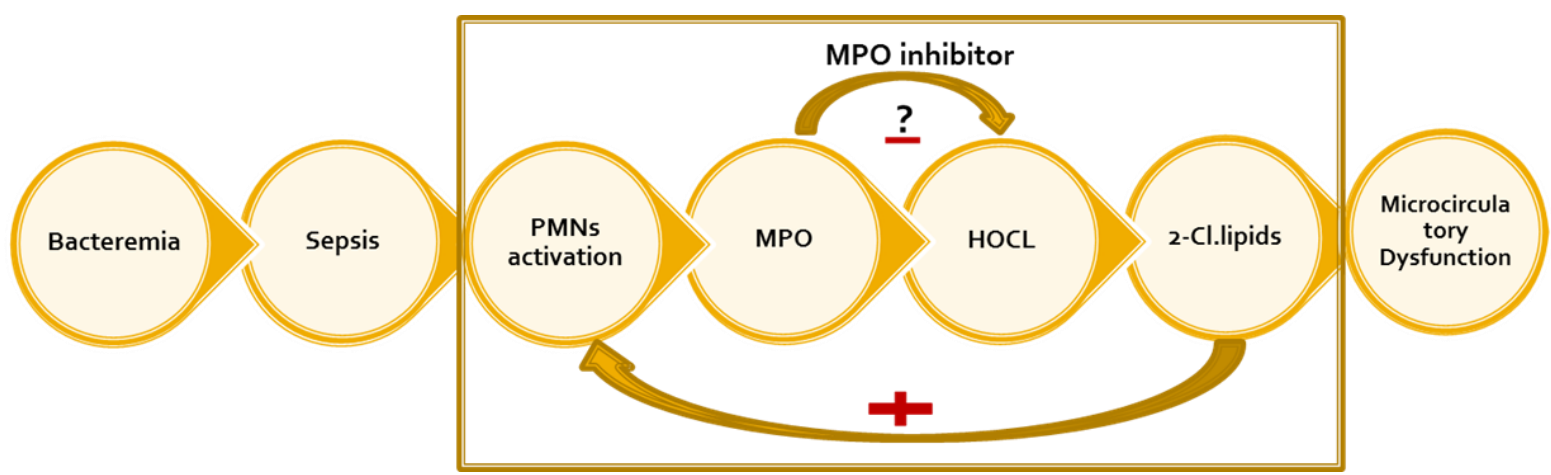

Figure 14. Hypothesis of 2-Cl. lipids elicit inflammation via positive feedback in sepsis.

In conclusion, chlorinated lipid induce a number of inflammatory responses in rat mesentery, including increased neutrophil/endothelial cell interactions, mast cell degranulation, ROS production and disrupted endothelial barrier. On the contrary, nonchlorinated lipid did not produce these responses. Future studies will endeavor to determine the targets of the different chlorinated lipid and/or specific receptors, identify underlying inflammatory mechanisms and determine whether these compounds could serve as reliable biomarkers and mediators for diseases likely to involve production of these lipid mediators such as sepsis or ischemia/reperfusion. 


\section{BIBLIOGRAPHY}

1. Connick RE. 1947. The interaction of hydrogen peroxide and hypochlorous acid in acidic solutions containing chloride ion. Journal of the American Chemical Society 69:1509-14

2. Levinsky R. 1980. The Neutrophil: Function and Clinical Disorders. Journal of medical genetics 17:160

3. Albert CJ, Crowley JR, Hsu F-F, Thukkani AK, Ford DA. 2001. Reactive Chlorinating Species Produced by Myeloperoxidase Target the Vinyl Ether Bond of Plasmalogens IDENTIFICATION OF 2-CHLOROHEXADECANAL. Journal of Biological Chemistry 276:23733-41

4. Wildsmith KR, Albert CJ, Anbukumar DS, Ford DA. 2006. Metabolism of myeloperoxidase-derived 2-chlorohexadecanal. Journal of Biological Chemistry 281:16849-60

5. De Backer D, Orbegozo Cortes D, Donadello K, Vincent J-L. 2014. Pathophysiology of microcirculatory dysfunction and the pathogenesis of septic shock. Virulence 5:73-9

6. Ford DA, Korthuis RJ, Hotchkiss RS, Albert CJ, McHowat J. 2016. Chlorinated Lipids are Potential Mediators of Microcirculatory Dysfunction During Sepsis. The FASEB Journal 30:653.2-.2

7. Klebanoff SJ. 2005. Myeloperoxidase: friend and foe. Journal of leukocyte biology 77:598-625

8. Klebanoff SJ, Waltersdorph AM, Rosen H. 1984. [52] Antimicrobial activity of myeloperoxidase. Methods in enzymology 105:399-403

9. Nathan C. 2006. Neutrophils and immunity: challenges and opportunities. Nature Reviews Immunology 6:173-82

10. Harrison J, Schultz J. 1976. Studies on the chlorinating activity of myeloperoxidase. Journal of Biological Chemistry 251:1371-4

11. Heinecke JW, Li W, Mueller DM, Bohrer A, Turk J. 1994. Cholesterol chlorohydrin synthesis by the myeloperoxidase-hydrogen peroxide-chloride system: potential markers for lipoproteins oxidatively damaged by phagocytes. Biochemistry 33:10127-36

12. Thomas EL, Jefferson MM, Grisham MB. 1982. Myeloperoxidase-catalyzed incorporation of amines into proteins: role of hypochlorous acid and dichloramines. Biochemistry 21:6299-308

13. Winterbourn CC, van den Berg JJ, Roitman E, Kuypers FA. 1992. Chlorohydrin formation from unsaturated fatty acids reacted with hypochlorous acid. Archives of biochemistry and biophysics 296:547-55

14. Pattison DI, Hawkins CL, Davies MJ. 2003. Hypochlorous acid-mediated oxidation of lipid components and antioxidants present in low-density lipoproteins: absolute rate constants, product analysis, and computational modeling. Chemical research in toxicology 16:439-49

15. Hazen SL, Hsu FF, Duffin K, Heinecke JW. 1996. Molecular chlorine generated by the myeloperoxidase-hydrogen peroxide-chloride system of phagocytes converts low density lipoprotein cholesterol into a family of chlorinated sterols. Journal of Biological Chemistry 271:23080-8

16. Murphy EJ, Schapiro MB, Rapoport SI, Shetty HU. 2000. Phospholipid composition and levels are altered in Down syndrome brain. Brain research 867:9-18

17. Thukkani AK, Martinson BD, Albert CJ, Vogler GA, Ford DA. 2005. Neutrophilmediated accumulation of 2-ClHDA during myocardial infarction: 2-ClHDA-mediated myocardial injury. American Journal of Physiology-Heart and Circulatory Physiology 288:H2955-H64 
18. Thukkani AK, Hsu F-F, Crowley JR, Wysolmerski RB, Albert CJ, Ford DA. 2002. Reactive Chlorinating Species Produced during Neutrophil Activation Target Tissue Plasmalogens PRODUCTION OF THE CHEMOATTRACTANT, 2CHLOROHEXADECANAL. Journal of Biological Chemistry 277:3842-9

19. Nusshold C, Kollroser M, Köfeler H, Rechberger G, Reicher H, et al. 2010. Hypochlorite modification of sphingomyelin generates chlorinated lipid species that induce apoptosis and proteome alterations in dopaminergic PC12 neurons in vitro. Free Radical Biology and Medicine 48:1588-600

20. Marsche G, Heller R, Fauler G, Kovacevic A, Nuszkowski A, et al. 2004. 2Chlorohexadecanal Derived From Hypochlorite-Modified High-Density LipoproteinAssociated Plasmalogen Is a Natural Inhibitor of Endothelial Nitric Oxide Biosynthesis. Arteriosclerosis, thrombosis, and vascular biology 24:2302-6

21. Nagan N, Zoeller RA. 2001. Plasmalogens: biosynthesis and functions. Progress in lipid research 40:199-229

22. Gorgas K, Teigler A, Komljenovic D, Just WW. 2006. The ether lipid-deficient mouse: tracking down plasmalogen functions. Biochimica et Biophysica Acta (BBA)-Molecular Cell Research 1763:1511-26

23. Moser AB, Steinberg SJ, Watkins PA, Moser HW, Ramaswamy K, et al. 2011. Human and great ape red blood cells differ in plasmalogen levels and composition. Lipids in health and disease 10:101

24. Gross RW. 1984. High plasmalogen and arachidonic acid content of canine myocardial sarcolemma: a fast atom bombardment mass spectroscopic and gas chromatography-mass spectroscopic characterization. Biochemistry 23:158-65

25. Han X, Gross RW. 1994. Electrospray ionization mass spectroscopic analysis of human erythrocyte plasma membrane phospholipids. Proceedings of the National Academy of Sciences 91:10635-9

26. Malavolta M, Bocci F, Boselli E, Frega NG. 2004. Normal phase liquid chromatographyelectrospray ionization tandem mass spectrometry analysis of phospholipid molecular species in blood mononuclear cells: application to cystic fibrosis. Journal of Chromatography B 810:173-86

27. Vance JE. 1990. Lipoproteins secreted by cultured rat hepatocytes contain the antioxidant 1-alk-1-enyl-2-acylglycerophosphoethanolamine. Biochimica et Biophysica Acta (BBA)Lipids and Lipid Metabolism 1045:128-34

28. Gribble GW. 2003. The diversity of naturally produced organohalogens. Chemosphere 52:289-97

29. Arnhold J, Furtmüller PG, Obinger C. 2003. Redox properties of myeloperoxidase. Redox report 8:179-86

30. Ruf J, Carayon P. 2006. Structural and functional aspects of thyroid peroxidase. Archives of biochemistry and biophysics 445:269-77

31. Carr A, Vissers M, Domigan N, Winterbourn C. 1997. Modification of red cell membrane lipids by hypochlorous acid and haemolysis by preformed lipid chlorohydrins. Redox report 3:263-71

32. Anbukumar DS, Shornick LP, Albert CJ, Steward MM, Zoeller RA, et al. 2010. Chlorinated lipid species in activated human neutrophils: lipid metabolites of 2chlorohexadecanal. Journal of lipid research 51:1085-92

33. Messner MC, Albert CJ, Ford DA. 2008. 2-Chlorohexadecanal and 2-chlorohexadecanoic acid induce COX-2 expression in human coronary artery endothelial cells. Lipids 43:581

34. Üllen A, Fauler G, Bernhart E, Nusshold C, Reicher H, et al. 2012. Phloretin ameliorates 2-chlorohexadecanal-mediated brain microvascular endothelial cell dysfunction in vitro. Free Radical Biology and Medicine 53:1770-81 
35. Thukkani AK, Albert CJ, Wildsmith KR, Messner MC, Martinson BD, et al. 2003. Myeloperoxidase-derived reactive chlorinating species from human monocytes target plasmalogens in low density lipoprotein. Journal of Biological Chemistry 278:36365-72

36. Thukkani AK, McHowat J, Hsu F-F, Brennan M-L, Hazen SL, Ford DA. 2003. Identification of $\alpha$-chloro fatty aldehydes and unsaturated lysophosphatidylcholine molecular species in human atherosclerotic lesions. Circulation 108:3128-33

37. Dever G, Stewart L-J, Pitt AR, Spickett CM. 2003. Phospholipid chlorohydrins cause ATP depletion and toxicity in human myeloid cells. FEBS letters 540:245-50

38. Dever G, Wainwright CL, Kennedy S, Spickett CM. 2006. Fatty acid and phospholipid chlorohydrins cause cell stress and endothelial adhesion.

39. Resch U, Semlitsch M, Hammer A, Susani-Etzerodt H, Walczak H, et al. 2011. Hypochlorite-modified low-density lipoprotein induces the apoptotic machinery in Jurkat T-cell lines. Biochemical and biophysical research communications 410:895-900

40. Wang W-y, Albert CJ, Ford DA. 2014. Alpha-Chlorofatty Acid Accumulates in Activated Monocytes and Causes Apoptosis Through Reactive Oxygen Species Production and Endoplasmic Reticulum StressSignificance. Arteriosclerosis, thrombosis, and vascular biology 34:526-32

41. Ince C. 2005. The microcirculation is the motor of sepsis. Critical care 9:S13

42. Opal S, Poll T. 2015. Endothelial barrier dysfunction in septic shock. Journal of internal medicine 277:277-93

43. Lam C, Tyml K, Martin C, Sibbald W. 1994. Microvascular perfusion is impaired in a rat model of normotensive sepsis. Journal of Clinical Investigation 94:2077

44. Aird WC. 2004. Endothelium as an organ system. Critical care medicine 32:S271-S9

45. Liu L-m, Dubick MA. 2005. Hemorrhagic shock-induced vascular hyporeactivity in the rat: relationship to gene expression of nitric oxide synthase, endothelin-1, and select cytokines in corresponding organs. Journal of Surgical Research 125:128-36

46. van Meurs M, Wulfert FM, Knol AJ, De Haes A, Houwertjes M, et al. 2008. Early organspecific endothelial activation during hemorrhagic shock and resuscitation. Shock 29:2919

47. Jordan JE, Zhao Z-Q, Vinten-Johansen J. 1999. The role of neutrophils in myocardial ischemia-reperfusion injury. Cardiovascular research 43:860-78

48. Lidington D, Ouellette Y, Tyml K. 2000. Endotoxin increases intercellular resistance in microvascular endothelial cells by a tyrosine kinase pathway. Journal of cellular physiology 185:117-25

49. Lidington D, Ouellette Y, Li F, Tyml K. 2003. Conducted vasoconstriction is reduced in a mouse model of sepsis. Journal of vascular research 40:149-58

50. Tyml K, Wang X, Lidington D, Ouellette Y. 2001. Lipopolysaccharide reduces intercellular coupling in vitro and arteriolar conducted response in vivo. American Journal of Physiology-Heart and Circulatory Physiology 281:H1397-H406

51. Zuidema MY KR. 2015. INtravital microscopic methods to evaluate anti-inflammatory effects and signaling mechanisms evoked by hydrogen sulfide. Methods Enzymol 555:93125

52. Proebstl D, Voisin M-B, Woodfin A, Whiteford J, D’Acquisto F, et al. 2012. Pericytes support neutrophil subendothelial cell crawling and breaching of venular walls in vivo. Journal of Experimental Medicine:jem. 20111622

53. Stark K, Eckart A, Haidari S, Tirniceriu A, Lorenz M, et al. 2013. Capillary and arteriolar pericytes attract innate leukocytes exiting through venules and'instruct'them with patternrecognition and motility programs. Nature immunology 14:41-51

54. Voisin M-B, Pröbstl D, Nourshargh S. 2010. Venular basement membranes ubiquitously express matrix protein low-expression regions: characterization in multiple tissues and remodeling during inflammation. The American journal of pathology 176:482-95 
55. Nauseef WM. 2007. How human neutrophils kill and degrade microbes: an integrated view. Immunological reviews 219:88-102

56. von Köckritz-Blickwede M, Nizet V. 2009. Innate immunity turned inside-out: antimicrobial defense by phagocyte extracellular traps. Journal of Molecular Medicine $87: 775-83$

57. Brinkmann V, Zychlinsky A. 2007. Beneficial suicide: why neutrophils die to make NETs. Nature Reviews Microbiology 5:577-82

58. Brinkmann V, Reichard U, Goosmann C, Fauler B, Uhlemann Y, et al. 2004. Neutrophil extracellular traps kill bacteria. science 303:1532-5

59. Akong-Moore K, Chow OA, von Köckritz-Blickwede M, Nizet V. 2012. Influences of chloride and hypochlorite on neutrophil extracellular trap formation. PLoS One 7:e42984

60. Wang Y, Thorlacius H. 2005. Mast cell-derived tumour necrosis factor- $\alpha$ mediates macrophage inflammatory protein-2-induced recruitment of neutrophils in mice. British journal of pharmacology 145:1062-8

61. Molderings G. 2010. Mast cell function in physiology and pathophysiology. Biotrend reviews 5:1-9

62. Weller CL, Collington SJ, Williams T, Lamb JR. 2011. Mast cells in health and disease. Clinical science 120:473-84

63. Caughey GH. 2007. Mast cell tryptases and chymases in inflammation and host defense. Immunological reviews 217:141-54

64. Sena LA, Chandel NS. 2012. Physiological roles of mitochondrial reactive oxygen species. Molecular cell 48:158-67

65. Kalinina E, Chernov N, Novichkova M. 2014. Role of glutathione, glutathione transferase, and glutaredoxin in regulation of redox-dependent processes. Biochemistry (Moscow) 79:1562-83

66. Cosentino-Gomes D, Rocco-Machado N, Meyer-Fernandes JR. 2012. Cell signaling through protein kinase $\mathrm{C}$ oxidation and activation. International journal of molecular sciences 13:10697-721

67. Wenk P. 2012. Mast cells. Mikro Graf 41:4-6

68. Gringhuis SI, Papendrecht-van der Voort EA, Leow A, Levarht EN, Breedveld FC, Verweij CL. 2002. Effect of redox balance alterations on cellular localization of LAT and downstream T-cell receptor signaling pathways. Molecular and cellular biology 22:40011

69. Hughes MR, McNagny KM. 2015. Mast Cells: Methods and Protocols. Humana Press

70. Claesson-Welsh L. 2015. Vascular permeability - the essentials. Upsala journal of medical sciences 120:135-43

71. Majno G, Shea SM, Leventhal M. 1969. Endothelial contraction induced by histaminetype mediators: an electron microscopic study. The Journal of cell biology 42:647

72. Zuidema MY, Yang Y, Wang M, Kalogeris T, Liu Y, et al. 2010. Antecedent hydrogen sulfide elicits an anti-inflammatory phenotype in postischemic murine small intestine: role of BK channels. American Journal of Physiology-Heart and Circulatory Physiology 299:H1554-H67

73. Zuidema MY, Peyton KJ, Fay WP, Durante W, Korthuis RJ. 2011. Antecedent hydrogen sulfide elicits an anti-inflammatory phenotype in postischemic murine small intestine: role of heme oxygenase-1. American Journal of Physiology-Heart and Circulatory Physiology 301:H888-H94

74. Gaskin FS, Kamada K, Zuidema MY, Jones AW, Rubin LJ, Korthuis RJ. 2011. Isoformselective 5'-AMP-activated protein kinase-dependent preconditioning mechanisms to prevent postischemic leukocyte-endothelial cell adhesive interactions. American Journal of Physiology-Heart and Circulatory Physiology 300:H1352-H60 
75. Steiner DR, Gonzalez NC, Wood JG. 2003. Mast cells mediate the microvascular inflammatory response to systemic hypoxia. Journal of Applied Physiology 94:325-34

76. Liu Y, Kalogeris T, Wang M, Zuidema MY, Wang Q, et al. 2012. Hydrogen sulfide preconditioning or neutrophil depletion attenuates ischemia-reperfusion-induced mitochondrial dysfunction in rat small intestine. American Journal of PhysiologyGastrointestinal and Liver Physiology 302:G44-G54

77. Feinman R, Deitch EA, Watkins AC, Abungu B, Colorado I, et al. 2010. HIF-1 mediates pathogenic inflammatory responses to intestinal ischemia-reperfusion injury. American Journal of Physiology-Gastrointestinal and Liver Physiology 299:G833-G43

78. Breslin JW, Sun H, Xu W, Rodarte C, Moy AB, et al. 2006. Involvement of ROCKmediated endothelial tension development in neutrophil-stimulated microvascular leakage. American journal of physiology. Heart and circulatory physiology 290:H741

79. Yuan Y, Chilian WM, Granger HJ, Zawieja DC. 1993. Permeability to albumin in isolated coronary venules. American Journal of Physiology-Heart and Circulatory Physiology 265:H543-H52

80. Aman J, Weijers EM, van Nieuw Amerongen GP, Malik AB, van Hinsbergh VW. 2016. Using cultured endothelial cells to study endothelial barrier dysfunction: Challenges and opportunities. American Journal of Physiology-Lung Cellular and Molecular Physiology 311:L453-L66

81. Boehme MW, Galle P, Stremmel W. 2002. Kinetics of thrombomodulin release and endothelial cell injury by neutrophil-derived proteases and oxygen radicals. Immunology 107:340-9

82. Ieni A, Barresi V, Branca G, Alberto Caruso R, Tuccari G. 2016. Mast Cell Interaction with Neutrophils in Human Gastric Carcinomas: Ultrastructural Observations. Analytical Cellular Pathology 2016

83. Yu Y, Blokhuis BR, Garssen J, Redegeld FA. 2016. Non-IgE mediated mast cell activation. European journal of pharmacology 778:33-43

84. Ranieri V, Rubenfeld G, Thompson B, Ferguson N, Caldwell E, et al. Acute respiratory distress syndrome: the Berlin Definition. JAMA: the journal of the American Medical Association. 2012; 307 (23): 2526-33, Epub 2012/07/17

85. Bengalli R, Mantecca P, Camatini M, Gualtieri M. 2012. Effect of nanoparticles and environmental particles on a cocultures model of the air-blood barrier. BioMed research international 2013

86. Bhattacharya J, Matthay MA. 2013. Regulation and repair of the alveolar-capillary barrier in acute lung injury. Annual review of physiology 75:593-615

87. Bichsel CA, Hall SR, Schmid RA, Guenat OT, Geiser T. 2015. Primary human lung pericytes support and stabilize in vitro perfusable microvessels. Tissue Engineering Part A 21:2166-76

88. Weiss SJ, Klein R, Slivka A, Wei M. 1982. Chlorination of taurine by human neutrophils: evidence for hypochlorous acid generation. Journal of Clinical Investigation 70:598

89. Davis GE, Senger DR. 2005. Endothelial extracellular matrix. Circulation research 97:1093-107

90. Davies PF. 1995. Flow-mediated endothelial mechanotransduction. Physiological reviews 75:519-60

91. Booth R, Noh S, Kim H. 2014. A multiple-channel, multiple-assay platform for characterization of full-range shear stress effects on vascular endothelial cells. Lab on a Chip 14:1880-90

92. Dejana E, Bazzoni G, Lampugnani MG. 1999. Vascular endothelial (VE)-cadherin: only an intercellular glue? Experimental cell research 252:13-9 
93. Orsenigo F, Giampietro C, Ferrari A, Corada M, Galaup A, et al. 2012. Phosphorylation of VE-cadherin is modulated by haemodynamic forces and contributes to the regulation of vascular permeability in vivo. Nature communications 3:1208

94. Gavard J, Gutkind JS. 2006. VEGF controls endothelial-cell permeability by promoting the $\beta$-arrestin-dependent endocytosis of VE-cadherin. Nature cell biology 8:1223-34

95. Kohn S, Nagy J, Dvorak H, Dvorak A. 1992. Pathways of macromolecular tracer transport across venules and small veins. Structural basis for the hyperpermeability of tumor blood vessels. Laboratory investigation; a journal of technical methods and pathology 67:596-607

96. Armulik A, Genové G, Mäe M, Nisancioglu MH, Wallgard E, et al. 2010. Pericytes regulate the blood-brain barrier. Nature 468:557-61

97. Dvorak A, Kohn S, Morgan ES, Fox P, Nagy JA, Dvorak HF. 1996. The vesiculovacuolar organelle (VVO): a distinct endothelial cell structure that provides a transcellular pathway for macromolecular extravasation. Journal of leukocyte biology 59:100-15

98. Adamson RH, Zeng M, Adamson GN, Lenz JF, Curry F. 2003. PAF-and bradykinininduced hyperpermeability of rat venules is independent of actin-myosin contraction. American Journal of Physiology-Heart and Circulatory Physiology 285:H406-H17

99. Waschke J, Drenckhahn D, Adamson R, Curry F. 2004. Role of adhesion and contraction in Rac 1-regulated endothelial barrier function in vivo and in vitro. American Journal of Physiology-Heart and Circulatory Physiology 287:H704-H11

100. Wojciak-Stothard B, Ridley AJ. 2002. Rho GTPases and the regulation of endothelial permeability. Vascular pharmacology 39:187-99

101. Baumer Y, Burger S, Curry F, Golenhofen N, Drenckhahn D, Waschke J. 2008. Differential role of Rho GTPases in endothelial barrier regulation dependent on endothelial cell origin. Histochemistry and cell biology 129:179-91

102. Boquet P, Lemichez E. 2003. Bacterial virulence factors targeting Rho GTPases: parasitism or symbiosis? Trends in cell biology 13:238-46

103. Waschke J, Baumgartner W, Adamson R, Zeng M, Aktories K, et al. 2004. Requirement of Rac activity for maintenance of capillary endothelial barrier properties. American Journal of Physiology-Heart and Circulatory Physiology 286:H394-H401

104. Fischmeister R. 2006. Is cAMP Good or Bad? : Am Heart Assoc

105. Mallat Z, Hugel B, Ohan J, Lesèche G, Freyssinet J-M, Tedgui A. 1999. Shed membrane microparticles with procoagulant potential in human atherosclerotic plaques. Circulation 99:348-53

106. Sugiyama S, Kugiyama K, Aikawa M, Nakamura S, Ogawa H, Libby P. 2004. Hypochlorous acid, a macrophage product, induces endothelial apoptosis and tissue factor expression. Arteriosclerosis, thrombosis, and vascular biology 24:1309-14

107. Dallegri F, Ottonello L, Ballestrero A, Bogliolo F, Ferrando F, Patrone F. 1990. Cytoprotection against neutrophil derived hypochlorous acid: a potential mechanism for the therapeutic action of 5-aminosalicylic acid in ulcerative colitis. Gut 31:184-6

108. Reber LL, Gillis CM, Starkl P, Jönsson F, Sibilano R, et al. 2017. Neutrophil myeloperoxidase diminishes the toxic effects and mortality induced by lipopolysaccharide. Journal of Experimental Medicine:jem. 20161238

109. El Kebir D, József L, Pan W, Filep JG. 2008. Myeloperoxidase delays neutrophil apoptosis through CD11b/CD18 integrins and prolongs inflammation. Circulation research 103:352-9

110. Johansson MW, Patarroyo M, Oberg F, Siegbahn A, Nilsson K. 1997. Myeloperoxidase mediates cell adhesion via the alpha M beta 2 integrin (Mac-1, CD11b/CD18). Journal of Cell Science 110:1133-9 
111. Klinke A, Nussbaum C, Kubala L, Friedrichs K, Rudolph TK, et al. 2011. Myeloperoxidase attracts neutrophils by physical forces. Blood 117:1350-8

112. Lau D, Mollnau H, Eiserich JP, Freeman BA, Daiber A, et al. 2005. Myeloperoxidase mediates neutrophil activation by association with CD11b/CD18 integrins. Proceedings of the National Academy of Sciences of the United States of America 102:431-6

113. Adam M, Gajdova S, Kolarova H, Kubala L, Lau D, et al. 2014. Red blood cells serve as intravascular carriers of myeloperoxidase. Journal of molecular and cellular cardiology 74:353-63

114. Venereau E, Casalgrandi M, Schiraldi M, Antoine DJ, Cattaneo A, et al. 2012. Mutually exclusive redox forms of HMGB1 promote cell recruitment or proinflammatory cytokine release. Journal of Experimental Medicine 209:1519-28

115. Yang H, Antoine DJ, Andersson U, Tracey KJ. 2013. The many faces of HMGB1: molecular structure-functional activity in inflammation, apoptosis, and chemotaxis.

Journal of leukocyte biology 93:865-73

116. Duerr MA, Aurora R, Ford DA. 2015. Identification of glutathione adducts of $\alpha$ chlorofatty aldehydes produced in activated neutrophils. Journal of lipid research 56:1014-24

117. Ford DA. 2015. Halogenated Lipids: Products of Peroxidase-Derived Reactive Halogenating Species Targeting Conventional Lipids. In Lipid Oxidation in Health and Disease:77-100: CRC Press. Number of 77-100 pp.

118. Wildsmith KR, Albert CJ, Hsu F-F, Kao JL-F, Ford DA. 2006. Myeloperoxidase-derived 2-chlorohexadecanal forms Schiff bases with primary amines of ethanolamine glycerophospholipids and lysine. Chemistry and physics of lipids 139:157-70

119. Wang J, Kubes P. 2016. A reservoir of mature cavity macrophages that can rapidly invade visceral organs to affect tissue repair. Cell 165:668-78 